\title{
RERTR-13 Irradiation Summary Report
}

D. M. Perez

M. A. Lillo

G. S. Chang

D. M. Wachs

G. A. Roth

N. E. Woolstenhulme

September 2012

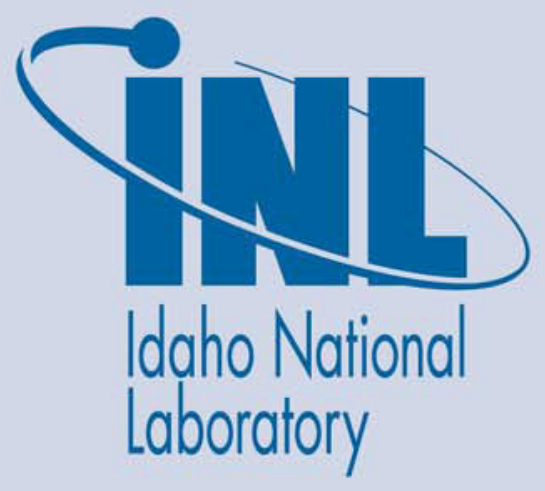

The INL is a U.S. Department of Energy National Laboratory operated by Battelle Energy Alliance 


\section{DISCLAIMER}

This information was prepared as an account of work sponsored by an agency of the U.S. Government. Neither the U.S. Government nor any agency thereof, nor any of their employees, makes any warranty, expressed or implied, or assumes any legal liability or responsibility for the accuracy, completeness, or usefulness, of any information, apparatus, product, or process disclosed, or represents that its use would not infringe privately owned rights. References herein to any specific commercial product, process, or service by trade name, trade mark, manufacturer, or otherwise, does not necessarily constitute or imply its endorsement, recommendation, or favoring by the U.S. Government or any agency thereof. The views and opinions of authors expressed herein do not necessarily state or reflect those of the U.S. Government or any agency thereof. 


\title{
RERTR-13 Irradiation Summary Report
}

\author{
D. M. Perez, M. A. Lillo, G. S. Chang,
}

D. M. Wachs, G. A. Roth, and

N. E. Woolstenhulme

September 2012

Idaho National Laboratory Idaho Falls, Idaho 83415

http://www.inl.gov

Prepared for the

U.S. Department of Energy

Office of National Nuclear Security

Administration

Under DOE Idaho Operations Office

Contract DE-AC07-05ID14517 


\title{
RERTR-13 Irradiation Summary Report
}

\author{
INL/EXT-12-27095
}

Revision 0

September 2012

Approved by:

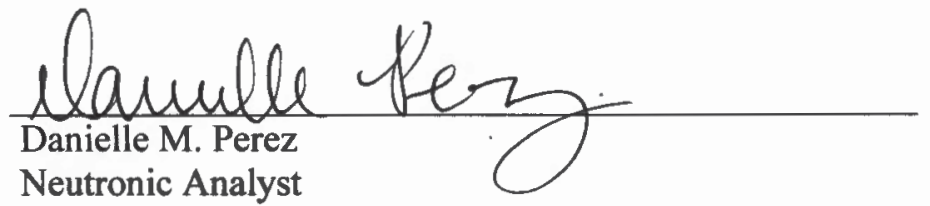

9/11/2012

Date

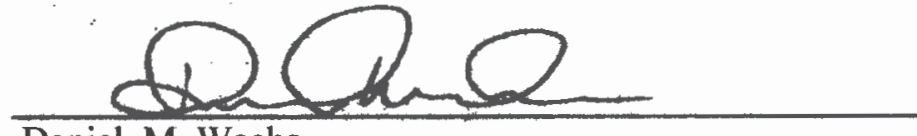

Daniel. M. Wachs

Principal Investigator

\begin{tabular}{c}
$9 / 11 / 2012$ \\
\hline Date \\
$9 / 11 / 2012$ \\
\hline Date
\end{tabular}

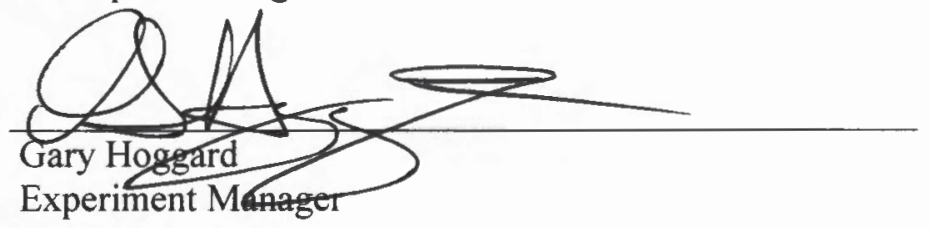

$\frac{9 / 11 / 2012}{\text { Date }}$




\section{SUMMARY}

The Reduced Enrichment for Research and Test Reactor (RERTR) experiment RERTR-13 was designed to assess performance of different types of neutron absorbers that can be potentially used as burnable poisons in the low enriched uranium-molybdenum based dispersion and monolithic fuels. ${ }^{1}$

The following report summarizes the life of the RERTR-13 experiment through end of irradiation, including as-run neutronic analysis results, thermal analysis results and hydraulic testing results. 


\section{CONTENTS}

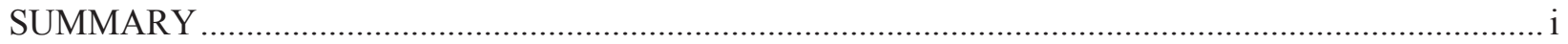

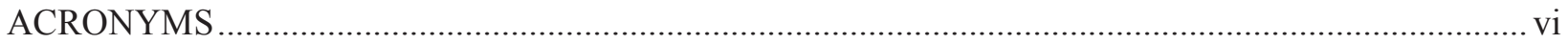

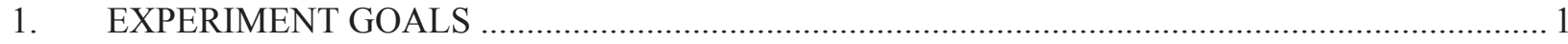

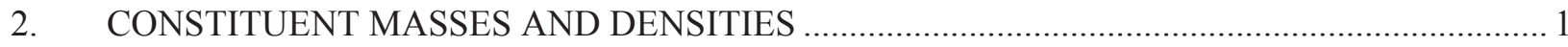

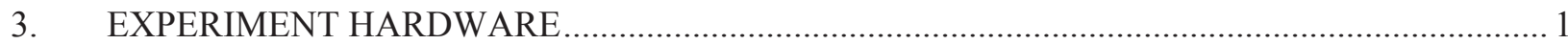

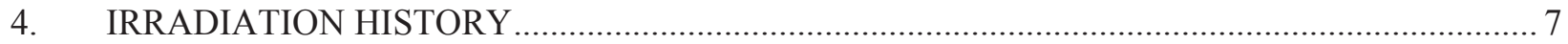

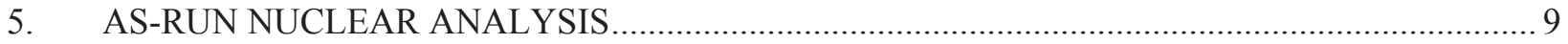

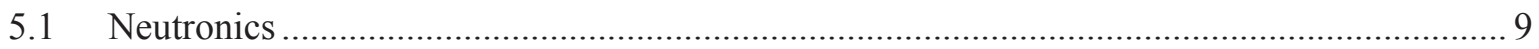

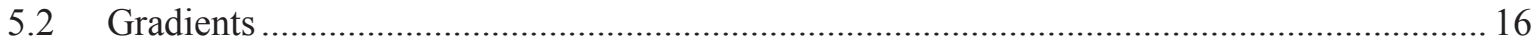

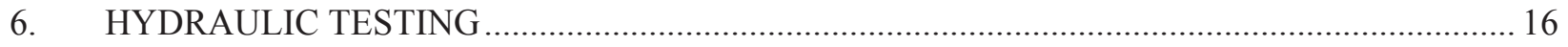

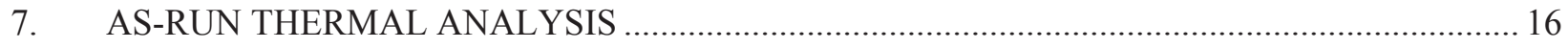

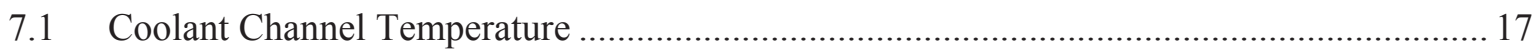

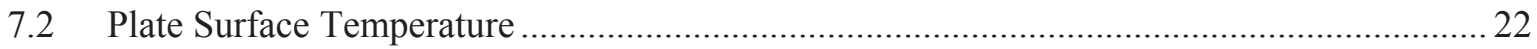

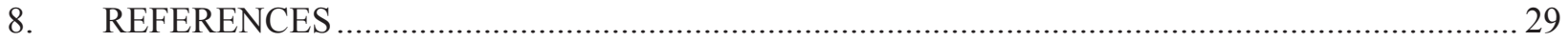

Appendix A Individual Plate Power and Fission Density Plots ........................................................... 30 


\section{FIGURES}

Figure 1: MCNP-Generated radial cross-section view of RERTR-13 test assembly (mini-plates

C1 through C4). NOTE: Plate IDs (not shown) face north. 1

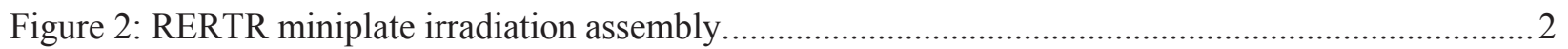

Figure 3: DWG-630244: RERTR monolithic fuel miniplate................................................................... 3

Figure 4: RERTR-13 detailed fuel plate sketch. ..................................................................................... 4

Figure 5: RERTR-13 detailed material plate sketch. ............................................................................. 5

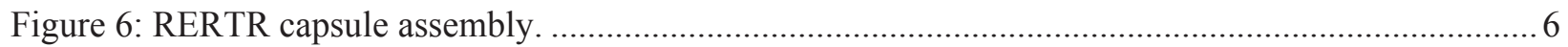

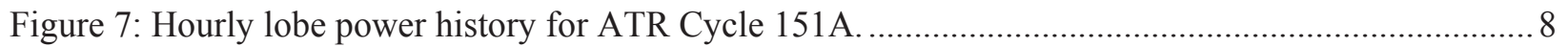

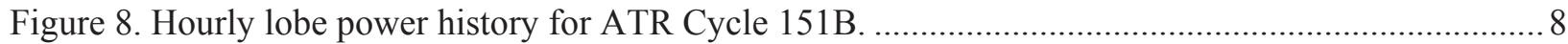

Figure 9: RERTR-13 capsule cross section with the plate IDs (not shown) and plate 1 facing north.

Figure 10: Coolant channel temperatures as a function of location along the RERTR-13 test assembly at BOC 151A (0.0 EFPD).

Figure 11: Coolant channel temperature as a function of location along the RERTR-13 test assembly at MOC1 151A (15.0 EFPD).

Figure 12: Coolant channel temperature as a function of location along the RERTR-13 test assembly at MOC2 151A (34.0 EFPD).

Figure 13: Coolant channel temperature as a function of location along the RERTR-13 test assembly at EOC 151A (56.1 EFPD).

Figure 14: Coolant channel temperature as a function of location along the RERTR-13 test assembly at BOC 151B (0.0 EFPD).

Figure 15: Coolant channel temperature as a function of location along the RERTR-13 test assembly at MOC1 151B (23.0 EFPD).

Figure 16: Coolant channel temperature as a function of location along the RERTR-13 test assembly at MOC2 151B (39.0 EFPD).

Figure 17: Coolant channel temperature as a function of location along the RERTR-13 test assembly at EOC 151B (51.3 EFPD). 


\section{TABLES}

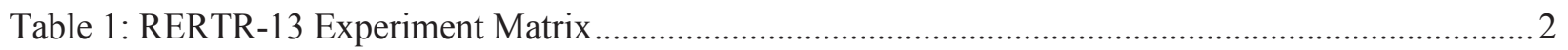

Table 2: RERTR-13 Capsule B nominal constituent masses and densities ${ }^{5}$.............................................. 1

Table 3: RERTR-13 capsule D nominal constituent masses and densities ${ }^{5}$. ............................................. 1

Table 4: RERTR Irradiation Hardware Drawing List............................................................................ 1

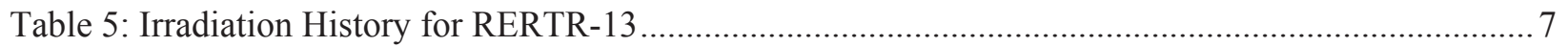

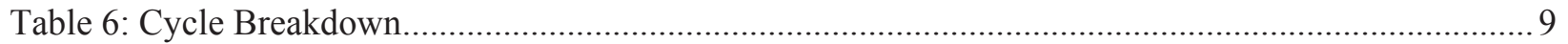

Table 7: Cycle 151A BOC As-Run HGR and Depletion Results for RERTR-13-1 in B-10 Scaled to As-Run Cycle Average East Source Power of 19.7 MW, 0EFPD ${ }^{5}$........................................ 10

Table 8: Cycle 151A MOC1 As-Run HGR and Depletion Results for RERTR-13-1 in B-10 Scaled to As-Run Cycle Average East Source Power of 19.7 MW, 15 EFPD ${ }^{5}$......................... 11

Table 9: Cycle 151A MOC2 As-Run HGR and Depletion Results for RERTR-13-1 in B-10 Scaled to As-Run Cycle Average East Source Power of 19.7 MW, 34 EFPD $^{5}$........................... 12

Table 10: Cycle 151A EOC As-Run HGR and Depletion Results for RERTR-13-1 in B-10 Scaled to As-Run Cycle Average East Source Power of 19.7 MW, 56.1 EFPD ${ }^{5}$.

Table 11: Cycle 151B BOC As-Run HGR and Depletion Results for RERTR-13-2 in B-10 Scaled to As-Run Cycle Average East Source Power of 19.9 MW, 0EFPD ${ }^{6}$.

Table 12: Cycle 151B MOC1 As-Run HGR and Depletion Results for RERTR-13-2 in B-10 Scaled to As-Run Cycle Average East Source Power of 19.9 MW, 23 EFPD ${ }^{6}$.....

Table 13: Cycle 151B MOC2 As-Run HGR and Depletion Results for RERTR-13-2 in B-10 Scaled to As-Run Cycle Average East Source Power of 19.9 MW, 39EFPD ${ }^{6}$.

Table 14: Cycle 151B EOC As-Run HGR and Depletion Results for RERTR-13-2 in B-10 Scaled to As-Run Cycle Average East Source Power of 19.9 MW, 51.3 EFPD ${ }^{6}$.

Table 15: Loss Coefficients for the RERTR Irradiation Test Vehicle Components ${ }^{7}$.....

Table 16: As-run minimum, maximum and average plate surface temperatures over fuel zone on the north side of the plate for capsules irradiated in Cycle 151A, BOC (0.0 EFPD)

Table 17: As-run minimum, maximum and average plate surface temperatures over fuel zone on the south side of the plate for capsules irradiated in Cycle 151A, BOC (0.0 EFPD) ....

Table 18: As-run minimum, maximum and average plate surface temperatures over fuel zone on the north side of the plate for capsules irradiated in Cycle 151A, MOC1 (15.0 EFPD)

Table 19: As-run minimum, maximum and average plate surface temperatures over fuel zone on the south side of the plate for capsules irradiated in Cycle 151A, MOC1 (15.0 EFPD)

Table 20: As-run minimum, maximum and average plate surface temperatures over fuel zone on the north side of the plate for capsules irradiated in Cycle 151A, MOC2 (34.0 EFPD)

Table 21: As-run minimum, maximum and average plate surface temperatures over fuel zone on the south side of the plate for capsules irradiated in Cycle 151A, MOC2 (34.0 EFPD)

Table 22: As-run minimum, maximum and average plate surface temperatures over fuel zone on the north side of the plate for capsules irradiated in Cycle 151A, EOC (56.1 EFPD).

Table 23: As-run minimum, maximum and average plate surface temperatures over fuel zone on the south side of the plate for capsules irradiated in Cycle 151A, EOC (56.1 EFPD) 
Table 24: As-run minimum, maximum and average plate surface temperatures over fuel zone on the north side of the plate for capsules irradiated in Cycle 151B, BOC (0.0 EFPD).

Table 25: As-run minimum, maximum and average plate surface temperatures over fuel zone on the south side of the plate for capsules irradiated in Cycle 151B, BOC (0.0 EFPD)

Table 26: As-run minimum, maximum and average plate surface temperatures over fuel zone on the north side of the plate for capsules irradiated in Cycle 151B, MOC1 (23.0 EFPD).

Table 27: As-run minimum, maximum and average plate surface temperatures over fuel zone on the south side of the plate for capsules irradiated in Cycle 151B, MOC1 (23.0 EFPD)

Table 28: As-run minimum, maximum and average plate surface temperatures over fuel zone on the north side of the plate for capsules irradiated in Cycle 151B, MOC2 (39.0 EFPD).

Table 29: As-run minimum, maximum and average plate surface temperatures over fuel zone on the south side of the plate for capsules irradiated in Cycle 151B, MOC2 (39.0 EFPD)

Table 30: As-run minimum, maximum and average plate surface temperatures over fuel zone on the north side of the plate for capsules irradiated in Cycle 151B, EOC (51.3 EFPD). .28

Table 31: As-run minimum, maximum and average plate surface temperatures over fuel zone on the south side of the plate for capsules irradiated in Cycle 151B, EOC (51.3 EFPD). 


\section{ACRONYMS}

$\begin{array}{ll}\text { Al } & \text { Aluminum } \\ \text { ATR } & \text { Advanced Test Reactor } \\ \text { BOC } & \text { Beginning of Cycle } \\ \text { DAS } & \text { Data Acquisition System } \\ \text { EFPD } & \text { Effective Full Power Days } \\ \text { EOC } & \text { End of Cycle } \\ \text { FD } & \text { Fuel Development } \\ \text { GTRI } & \text { Global Threat Reduction Initiative } \\ \text { HIP } & \text { Hot Isostatic Pressing } \\ \text { L2AR } & \text { Local-to-Average Ratio } \\ \text { LEU } & \text { Low Enriched Uranium } \\ \text { MCNP } & \text { Monte Carlo N-Particle } \\ \text { MOC } & \text { Middle of Cycle } \\ \text { Mo } & \text { Molybdenum } \\ \text { RERTR } & \text { Reduced Enrichment Research and Test Reactor } \\ \text { U } & \text { Uranium } \\ \text { U-Mo } & \text { Uranium-Molybdenum Alloy } \\ \text { Zr } & \text { Zirconium }\end{array}$




\section{RERTR-13 Irradiation Summary Report \\ 1. EXPERIMENT GOALS}

In support of the Global Threat Reduction Initiative (GTRI) Fuel Development (FD) program (historically known as Reduced Enrichment Research and Test Reactor (RERTR)), the RERTR-13 experiment was designed to assess performance of different types of neutron absorbers that can be potentially used as burnable poisons in the low enriched uranium-molybdenum based dispersion and monolithic fuels. ${ }^{1}$

The RERTR-13 test assembly holds 4 capsules, designated as A, B, C and D, with A at the top of the assembly and D at the bottom. Each capsule has 2 levels, with 4 plate positions per level, for a total of 8 plate positions per capsule and 32 plate positions per assembly. Within each capsule the 8 plate positions are azimuthally designated as 1 through 4 in the upper level and 5 through 8 in the lower level. The experiment matrix for the two irradiation cycles is shown in Table 1. The material plates in capsule B were irradiated during the first cycle then replaced with aluminum dummy plates for the second cycle. The miniplates in capsule D were irradiated over both cycles. The RERTR-13 mini-plates were oriented edge on to the core with the plate identification numbers facing North (see Figure 1).

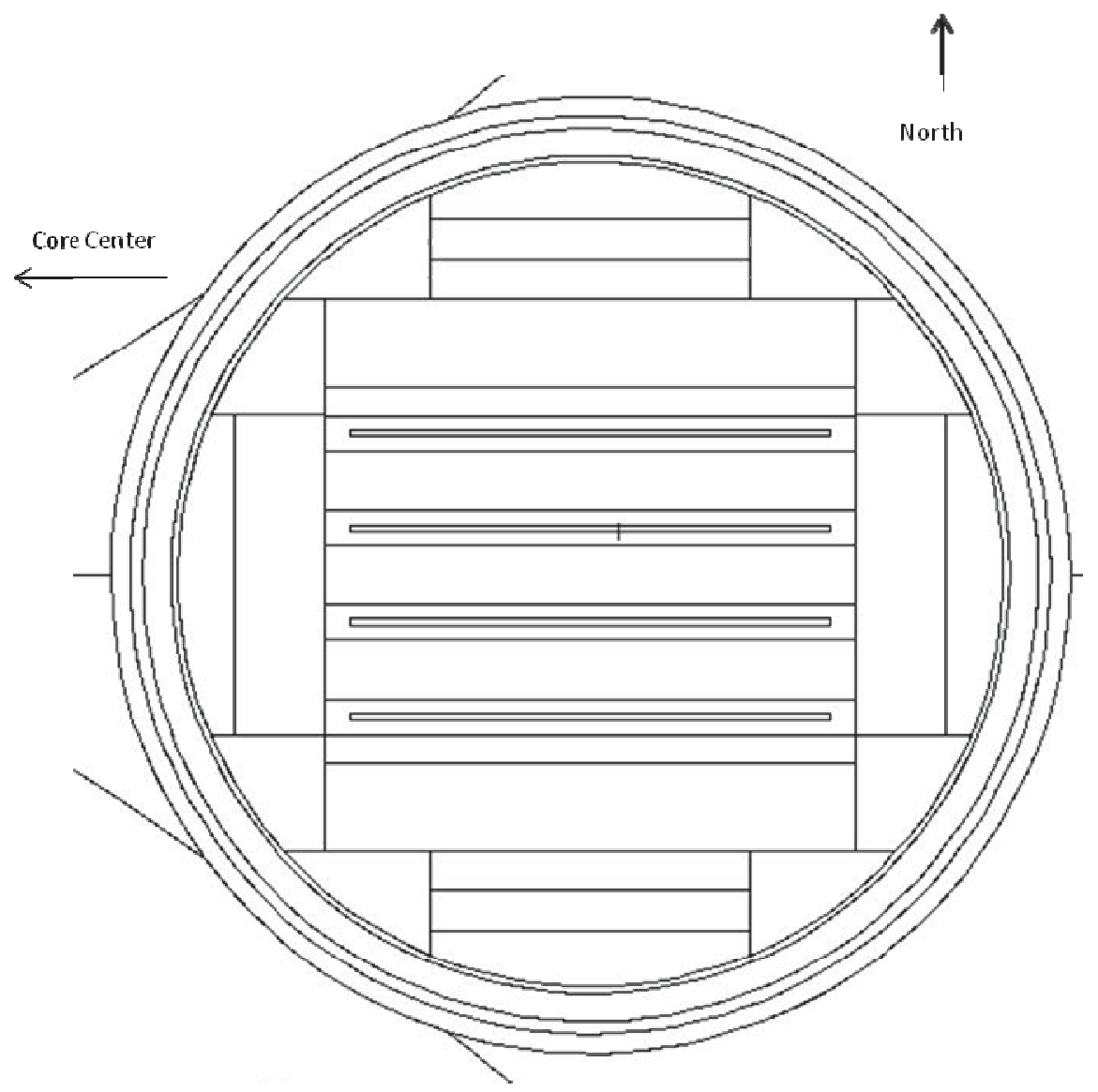

Figure 1: MCNP-Generated radial cross-section view of RERTR-13 test assembly (mini-plates C1 through C4). NOTE: Plate IDs (not shown) face north. 
Table 1: RERTR-13 Experiment Matrix

\begin{tabular}{|c|c|c|c|c|c|}
\hline ID & Capsule & Capsule Position 1 & Capsule Position 2 & Capsule Position 3 & Capsule Position 4 \\
\hline \multirow{4}{*}{ 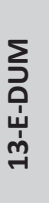 } & \multirow{2}{*}{ Top } & $\mathrm{A} 1$ & $\mathrm{~A} 2$ & A3 & A4 \\
\hline & & Aluminum Dummy & Aluminum Dummy & Aluminum Dummy & Aluminum Dummy \\
\hline & \multirow{2}{*}{ Bottom } & A5 & A6 & A7 & A8 \\
\hline & & Aluminum Dummy & Aluminum Dummy & Aluminum Dummy & Aluminum Dummy \\
\hline \multirow{4}{*}{$\begin{array}{l}\text { N} \\
\dot{\varphi} \\
\ddot{m}\end{array}$} & \multirow{2}{*}{ Top } & B1 & B2 & B3 & B4 \\
\hline & & Aluminum Dummy & Aluminum Dummy & Aluminum Dummy & Aluminum Dummy \\
\hline & \multirow[b]{2}{*}{ Bottom } & B5 & B6 & B7 & B8 \\
\hline & & $\begin{array}{c}\mathrm{B}_{4} \mathrm{C}-\mathrm{Al} \text { dispersion } \\
\text { (Natural B) } \\
\text { PCPOG4 }\end{array}$ & $\begin{array}{c}\text { Al-1.5B alloy } \\
\text { (Enriched B) } \\
\text { PAPOF0 } \\
\end{array}$ & $\begin{array}{c}\mathrm{ZrB}_{2} \text {-Al dispersion } \\
\text { (Enriched B) } \\
\text { PZP0E8 } \\
\end{array}$ & $\begin{array}{c}\mathrm{B}_{4} \mathrm{C}-\mathrm{Al} \text { dispersion } \\
\text { (Enriched B) } \\
\text { PCPOE7 }\end{array}$ \\
\hline \multirow{4}{*}{ 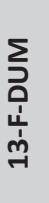 } & \multirow{2}{*}{ Top } & C1 & C2 & C3 & C4 \\
\hline & & Aluminum Dummy & Aluminum Dummy & Aluminum Dummy & Aluminum Dummy \\
\hline & \multirow{2}{*}{ Bottom } & $\mathrm{C5}$ & C6 & $\mathrm{C7}$ & C8 \\
\hline & & Aluminum Dummy & Aluminum Dummy & Aluminum Dummy & Aluminum Dummy \\
\hline \multirow{4}{*}{ ભ̣ } & & D1 & D2 & D3 & D4 \\
\hline & Top & $\begin{array}{c}\text { U-7Mo }+\mathrm{B}_{4} \mathrm{C} \\
\text { dispersion in Al-4\% Si, } \\
\text { U235-25\%, (Natural B ) } \\
\text { RCR3F1 }\end{array}$ & $\begin{array}{c}\text { U-7Mo }+\mathrm{B}_{4} \mathrm{C} \\
\text { dispersion in Al-4\% Si, } \\
\text { U235-69\%, (Natural B ) } \\
\text { RCR7F5 }\end{array}$ & $\begin{array}{c}\text { U-7Mo }+\mathrm{ZrB}_{2} \\
\text { dispersion in Al-4\% Si, } \\
\text { U235-69\%, (Natural B) } \\
\text { RZR7F7 }\end{array}$ & $\begin{array}{c}\text { U-7Mo }+\mathrm{ZrB}_{2} \\
\text { dispersion in Al-4\% Si, } \\
\text { U235-25\%, (Natural B) } \\
\text { RZR3F3 }\end{array}$ \\
\hline & & D5 & D6 & D7 & D8 \\
\hline & Bottom & $\begin{array}{c}\text { Al-4.5B alloy } \\
\text { (Enriched B) } \\
\text { PBPOF9 }\end{array}$ & $\begin{array}{l}\text { Al-1.5B alloy } \\
\text { (Enriched B) } \\
\text { PAP0G2 }\end{array}$ & $\begin{array}{c}\mathrm{ZrB}_{2} \text {-Al dispersion } \\
\text { (Enriched B) } \\
\text { PZPOE9 }\end{array}$ & $\begin{array}{c}\mathrm{B}_{4} \mathrm{C}-\mathrm{Al} \text { dispersion } \\
\text { (Enriched B) } \\
\text { PCP0D6 }\end{array}$ \\
\hline
\end{tabular}




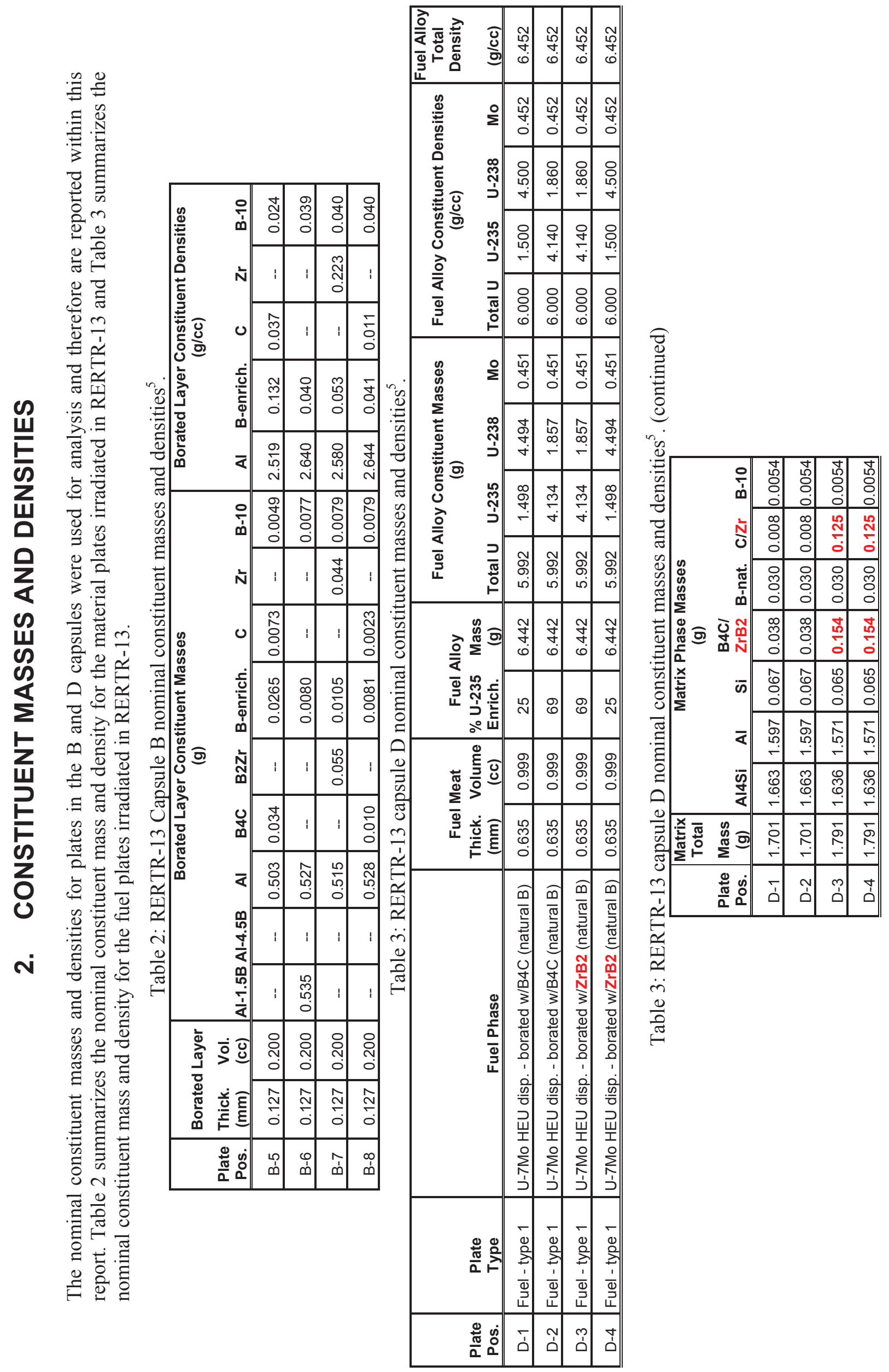




\section{EXPERIMENT HARDWARE}

The experiment hardware configuration is identical to that used in the RERTR-7A, $-7 \mathrm{~B},-8,-9 \mathrm{~A},-9 \mathrm{~B},-$ $10 \mathrm{~A}$ and $-10 \mathrm{~B}$ experiments. A list of irradiation hardware drawings used for analysis is given in Table 4.

Table 4: RERTR Irradiation Hardware Drawing List.

\begin{tabular}{cl}
\hline \multicolumn{1}{c}{$\begin{array}{c}\text { Drawing } \\
\text { Number }\end{array}$} & \multicolumn{1}{c}{ Drawing Title } \\
\hline DWG-630223 & RERTR ATR Large B-Position Irradiation Experiment Assembly \\
DWG-630233 & ATR Large B-Position Basket \\
DWG-630231 & ATR Top Spacer Assembly \\
DWG-630225 & ATR Upper Spacer Assembly \\
DWG-630229 & ATR Bottom Spacer Assembly \\
DWG-630227 & ATR Large B-Position Fuel Capsule Assembly \\
DWG-630237 & Fuel Capsule \\
DWG-630239 & Capsule Cap \\
DWG-630244 & RERTR Mini-Plate \\
\hline
\end{tabular}

The RERTR miniplate irradiation assembly, (see Figure 2) shows the main components of the test assembly, which include the bottom spacer, upper and top spacers, experiment capsules and basket. The bottom spacer elevates the experiment capsules to the correct location in the core. The upper and top spacers allow the operators to assure that the experiment is seated fully into the basket. All spacers are similar to the capsule design except the spacers do not have the grooves for the plates. The capsules hold the fuel plates; a capsule cap is welded onto the top of the capsule to keep the plates from sliding out during handling and irradiation. The miniplate drawing (DWG-630244) and RERTR miniplate capsule assembly are shown in Figure 3, Figure 4, Figure 5 and Figure 6, respectively. Each capsule has a notch at the top and a groove at the bottom which allow the capsules to stack and align properly into the core. The basket holds the test assembly in the reactor during irradiation, the notches on the outer wall allow for bypass coolant flow to cool the outer wall. The basket has two guide bars on the inside wall to guide the assembly into the baskets. 


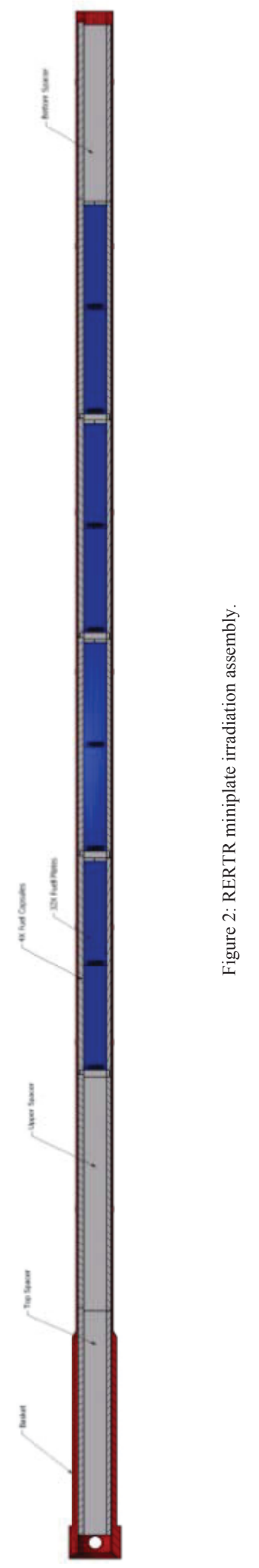




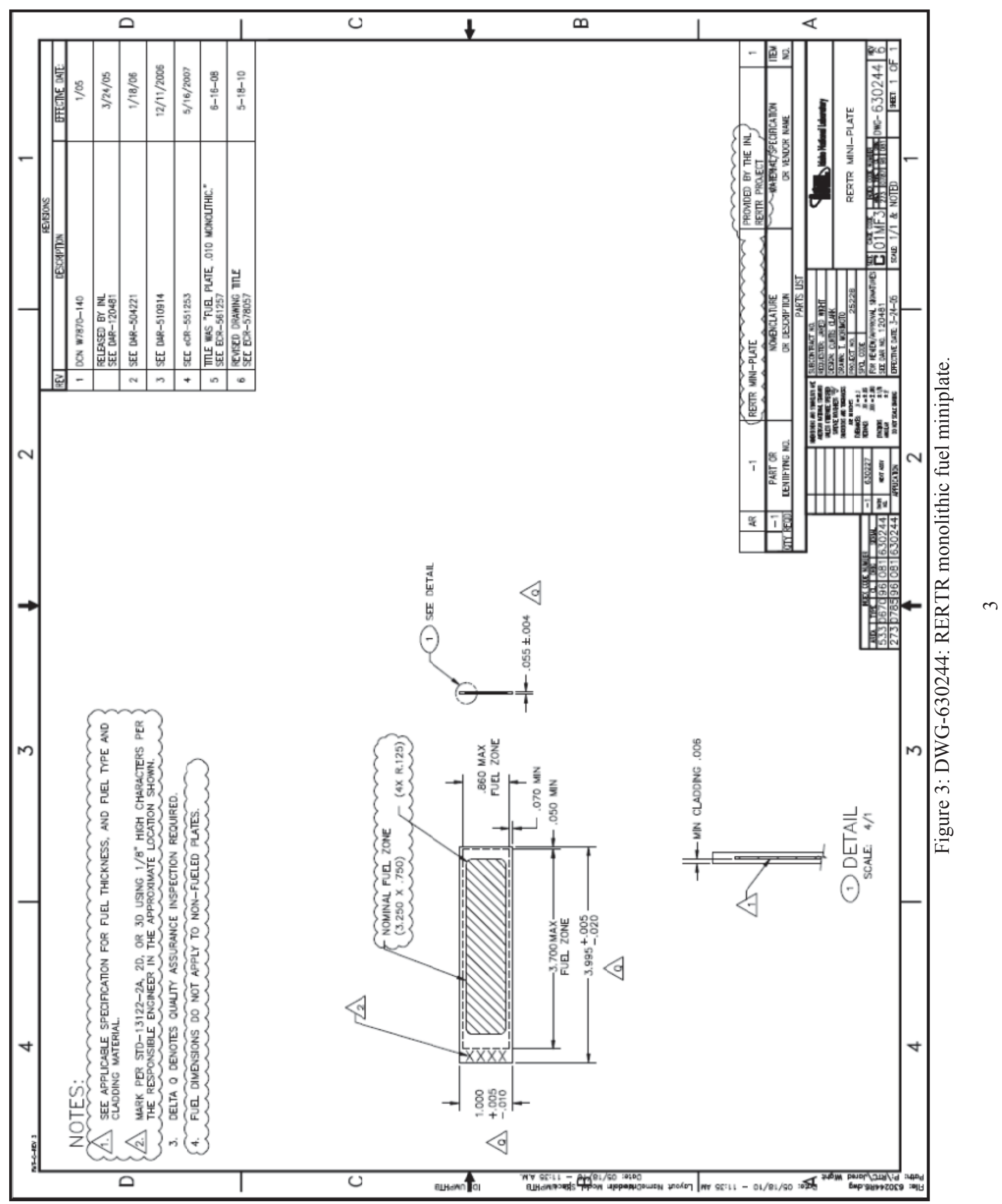



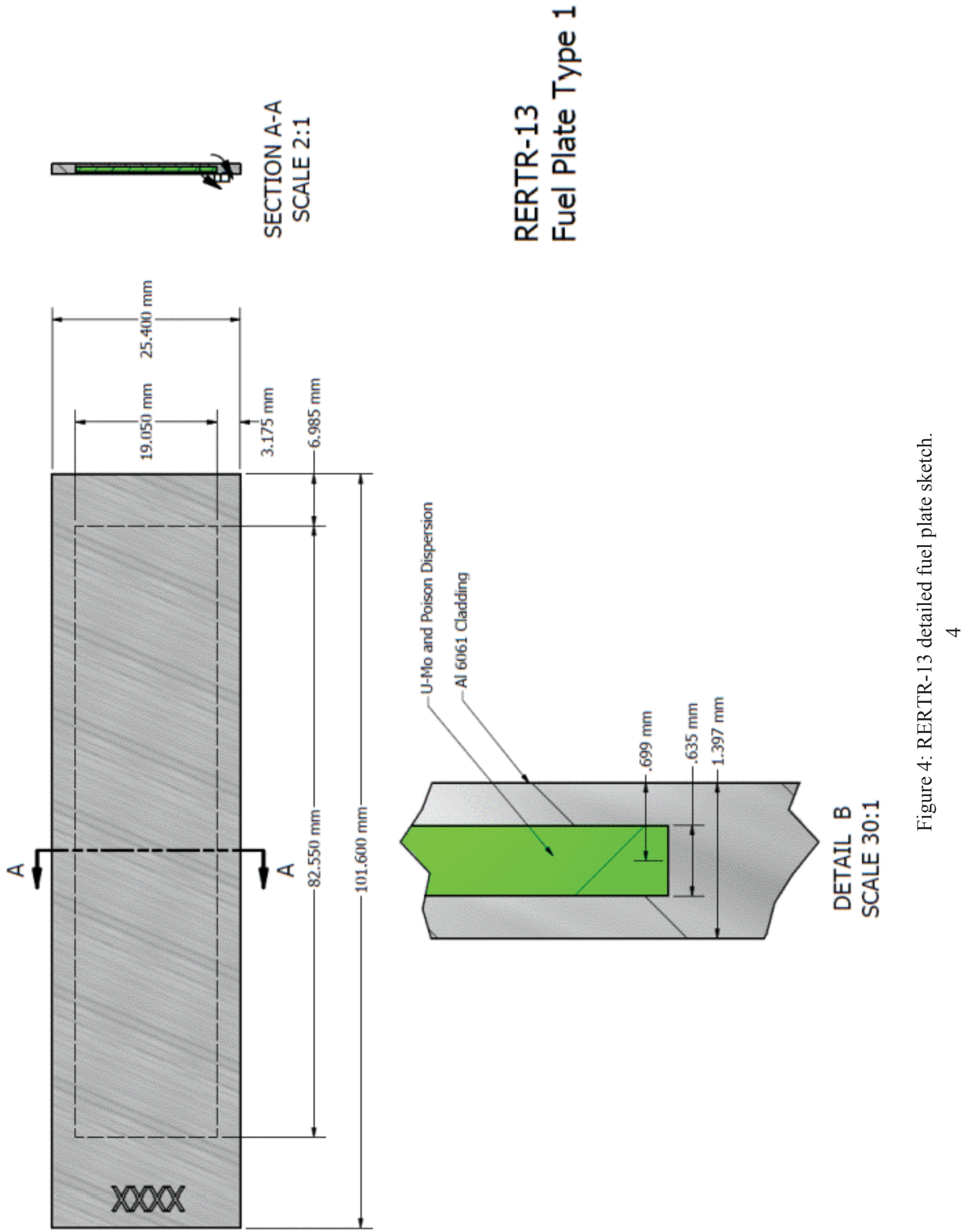


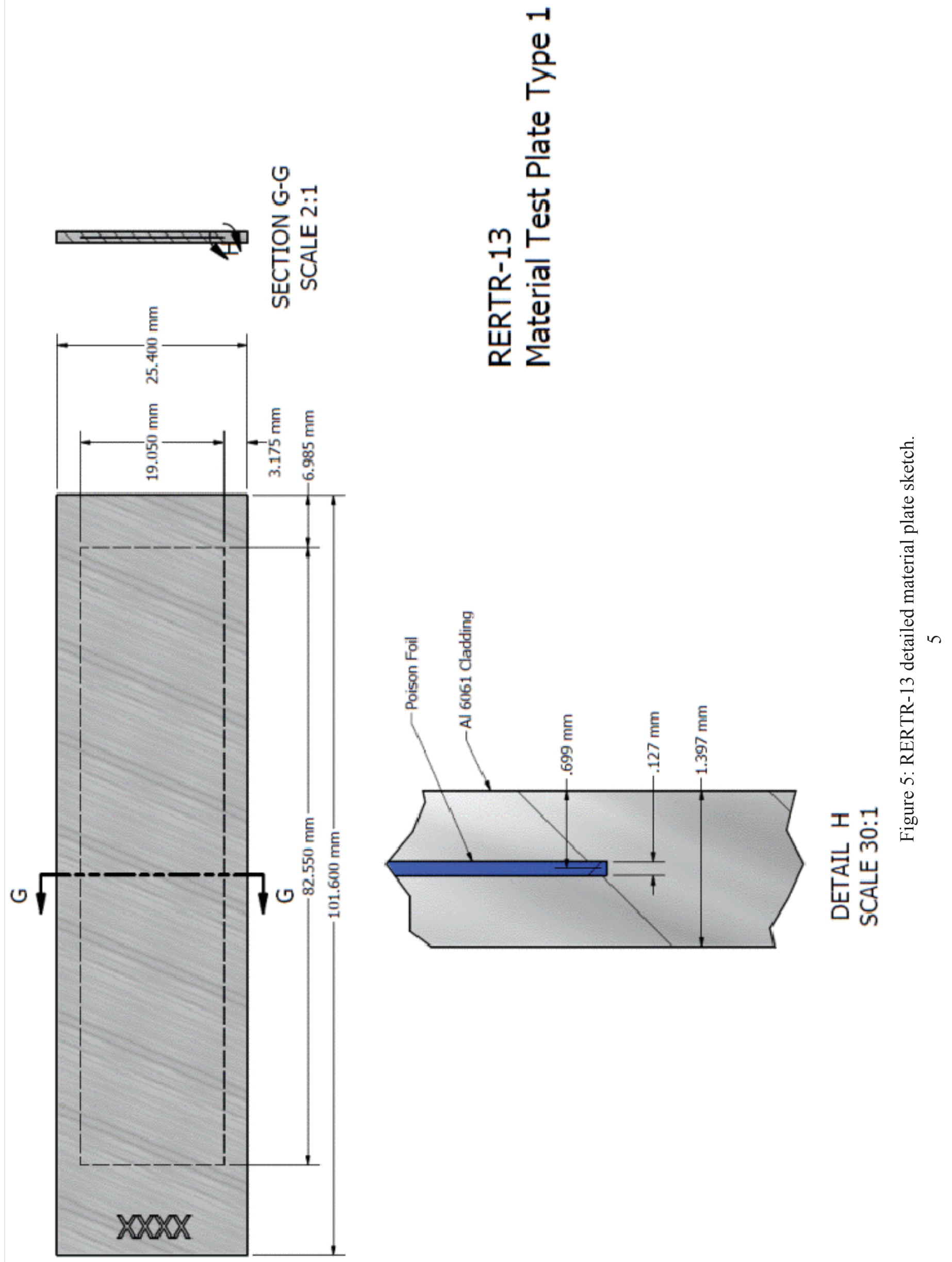




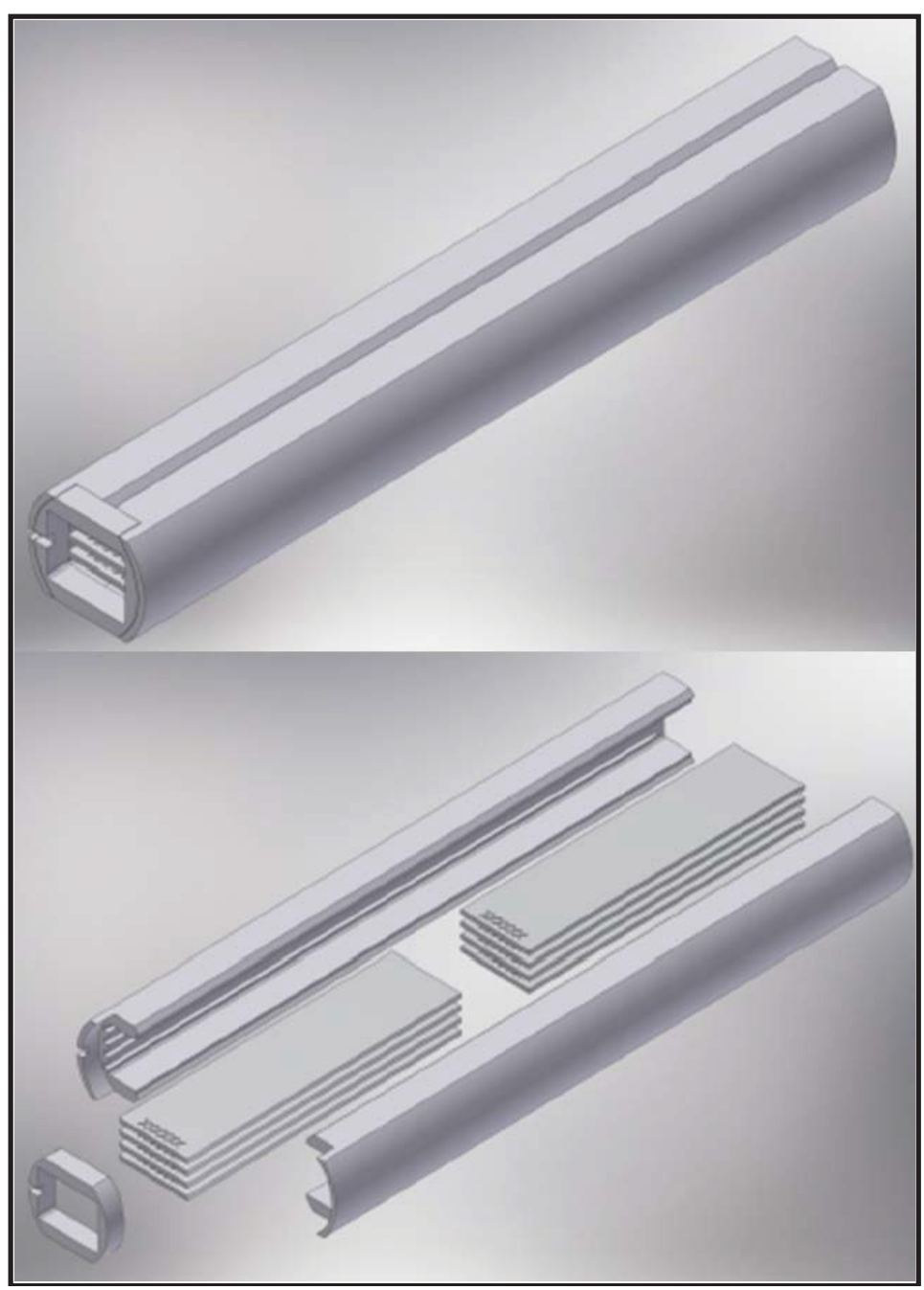

Figure 6: RERTR capsule assembly. 


\section{IRRADIATION HISTORY}

The RERTR-13 test assembly was irradiated in cycle 151A and cycle 151B. RERTR-13-1 and RERTR-13-2 were irradiated in the large-B position B-10. The power of position B-10 is represented by the east lobe power which is the average of the NE, $\mathrm{C}$ and $\mathrm{SE}$ lobe powers, $\mathrm{E}=(\mathrm{NE}+\mathrm{C}+\mathrm{SE}) / 3$. Cycle 151A ran for a total of 56.1 EFPDs at an average total core power of 101.7 MW (east lobe power of 19.7 MW) and cycle 151B ran for a total of 51.3 EFPDs at an average total core power of 101.5 MW (east lobe power of $19.9 \mathrm{MW})$.

There was one mid-cycle SCRAM during cycle 151A from with a duration of 3 days from 12/25/2011 12/28/2011. There were two mid-cycle SRAMs during cycle 151B from 3/22/2012 - 3/25/2012 and $3 / 27 / 2012-4 / 7 / 2012$, total duration of with a duration of 14 days. This information is tabulated in Table 5.

Table 5: Irradiation History for RERTR-13

\begin{tabular}{|c|c|c|c|c|c|c|}
\hline & RERTR-13 & & $\begin{array}{c}\text { Mid- } \\
\text { Cycle } \\
\text { Scram } \\
\text { Decay } \\
\text { Capsules } \\
\text { CYCLE }\end{array}$ & $\begin{array}{c}\text { East } \\
\text { Irradiated } \\
\text { Source } \\
\text { Power } \\
\text { (MW) }\end{array}$ & $\begin{array}{c}\text { Total } \\
\text { Core } \\
\text { Power } \\
\text { (MW) }\end{array}$ \\
\hline 151A & B, D & $\begin{array}{c}12 / 14 / 2011- \\
02 / 11 / 2012\end{array}$ & 56.1 & 3 & 19.7 & 101.7 \\
\hline 151B & D & $\begin{array}{c}03 / 01 / 2012- \\
05 / 05 / 2012\end{array}$ & 51.3 & 14 & 19.9 & 101.5 \\
\hline
\end{tabular}

The power history for each cycle is obtained as in ATR Surveillance Report from the ATR Data Acquisition System (DAS). The plots of each lobe power on an hourly basis are shown in Figure 7 and Figure 8 for cycle 151A and 151B, respectively. 


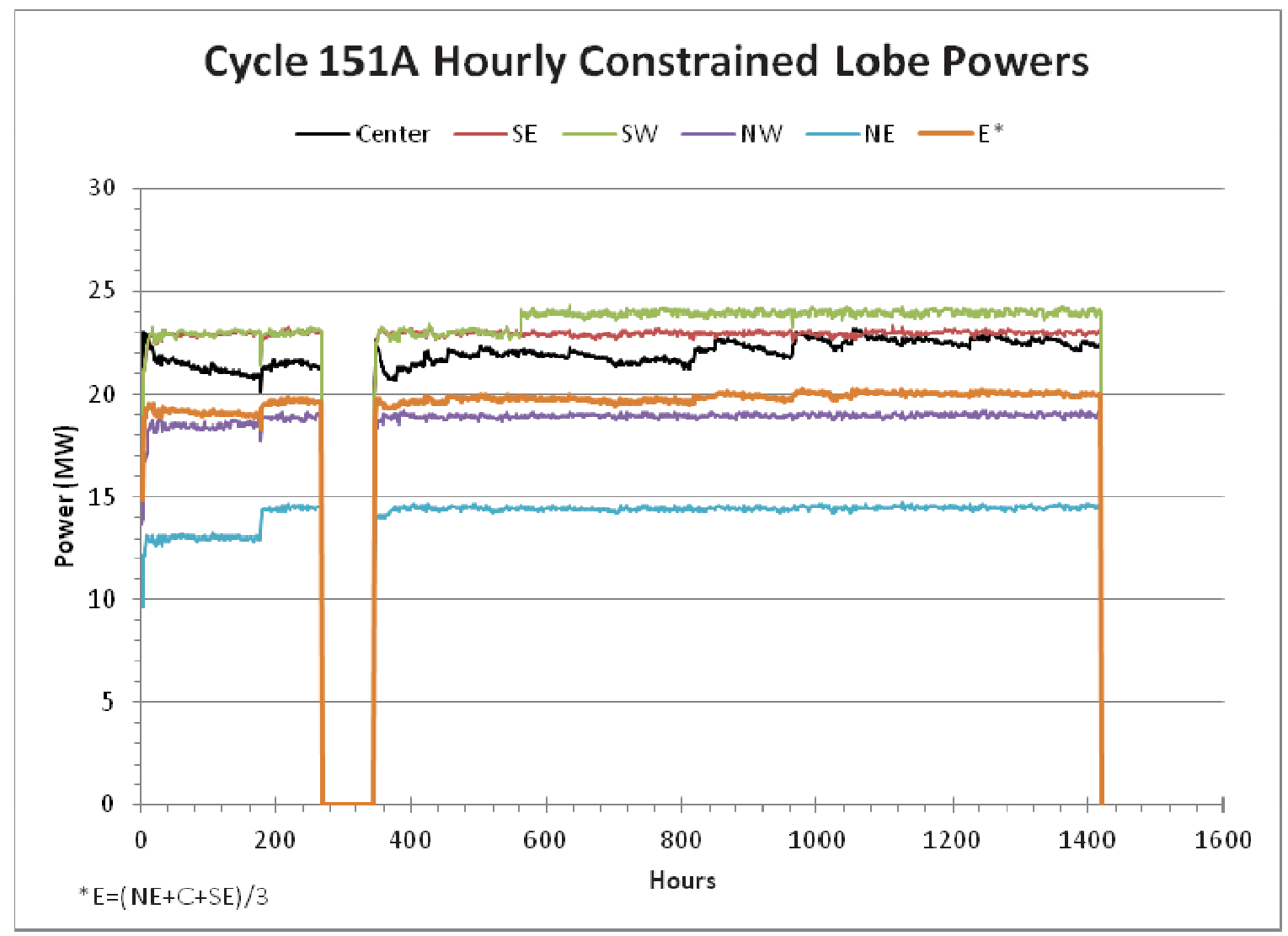

Figure 7: Hourly lobe power history for ATR Cycle 151A.

\section{Cycle 151B Hourly Constrained Lobe Powers}

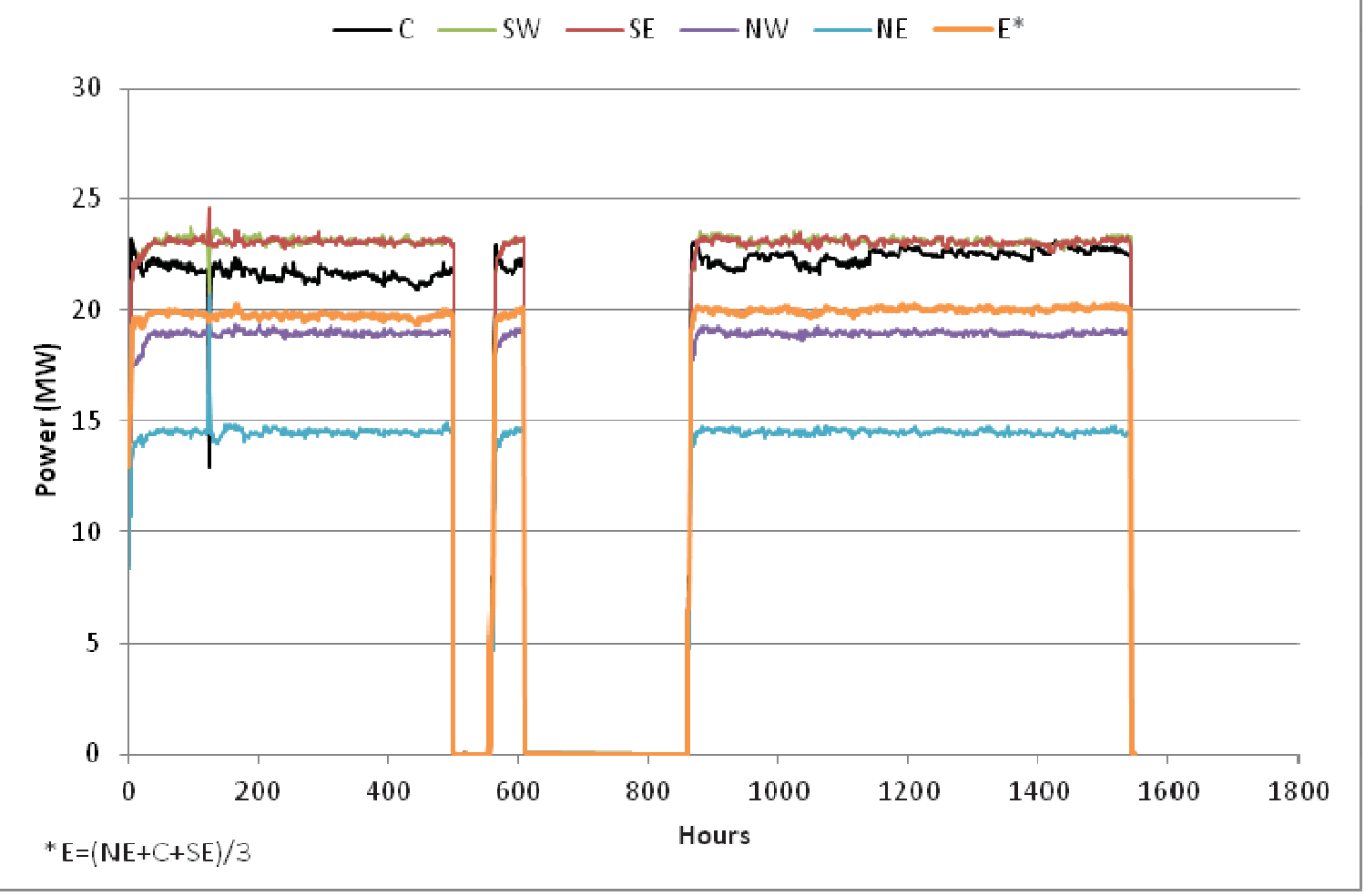

Figure 8. Hourly lobe power history for ATR Cycle 151B. 


\section{AS-RUN NUCLEAR ANALYSIS}

\section{$5.1 \quad$ Neutronics}

The as-run calculations were performed using the irradiation history in Table 5 and the Monte Carlo N-Particle (MCNP) code. The calculated as-run fission heat rates, fission densities, and asrun U-235 burnup results for the fueled miniplates reported have an uncertainty band $(1 \sigma)$ of $2.5 \%{ }^{5,6}$ The time intervals used to calculate the average plate power and burnup are shown in Table 6. The average plate power and burnup for the time intervals for cycle 151A are shown in Table 7 through Table 10. The average plate power and burnup for the time intervals for cycle 151B are shown in Table 11 through Table 14. The plots of the power and fission density as a function of the ATR Cycle time interval are presented in Appendix A.

Table 6: Cycle Breakdown

\begin{tabular}{ccc}
\hline Time Interval & $\begin{array}{c}151 \mathrm{~A} \\
\text { (days) }\end{array}$ & $\begin{array}{c}151 \mathrm{~B} \\
\text { (days) }\end{array}$ \\
\hline BOC & $1.00 \mathrm{E}-04$ & $1.00 \mathrm{E}-4$ \\
\hline MOC 1 & 15.0 & 23.0 \\
\hline MOC 2 & 19.0 & 16.0 \\
\hline EOC & 22.1 & 12.3 \\
\hline Total EFPDs & 56.1 & 51.3 \\
\hline Cumulative & 56.1 & 107.4 \\
\hline
\end{tabular}

The MCNP-calculated neutronic results reported were calculated using the nominal miniplates dimensions shown in section 2 . 
Table 7: Cycle 151A BOC As-Run HGR and Depletion Results for RERTR-13-1 in B-10 Scaled to As-Run Cycle Average East Source Power of 19.7 MW, 0EFPD 5 .

\begin{tabular}{|c|c|c|c|c|c|c|c|c|c|}
\hline \multirow[b]{2}{*}{$\begin{array}{c}\text { Capsule } \\
\text { ID }\end{array}$} & \multirow[b]{2}{*}{$\begin{array}{c}\text { Plate } \\
\text { Position }\end{array}$} & \multirow[b]{2}{*}{$\begin{array}{c}\text { Plate } \\
\text { ID }\end{array}$} & \multirow[b]{2}{*}{$\begin{array}{c}\text { BA/Fuel } \\
\text { Neutron } \\
\text { Flux } \\
\left(\mathrm{n} / \mathrm{cm}^{2}-\mathrm{sec}\right) \\
\end{array}$} & \multicolumn{2}{|c|}{ Burnable Absorber } & \multicolumn{4}{|c|}{ Fuel } \\
\hline & & & & $\begin{array}{c}\text { Power } \\
\text { Density } \\
\text { (W/cc) }\end{array}$ & \begin{tabular}{|c|}
$\%$ \\
Depletion \\
B-10 \\
$(\%)$ \\
\end{tabular} & $\begin{array}{l}\text { Fission } \\
\text { Power } \\
\text { Density } \\
\text { (W/cc) } \\
\end{array}$ & $\begin{array}{c}\text { Surface } \\
\text { Heat } \\
\text { Flux } \\
\left(\mathrm{W} / \mathrm{cm}^{2}\right)\end{array}$ & $\begin{array}{c}\text { Fission } \\
\text { Density } \\
\text { (fissions/cc) }\end{array}$ & \begin{tabular}{|c|}
$\%$ \\
Depletion \\
U-235 \\
$(\%)$ \\
\end{tabular} \\
\hline \multirow{8}{*}{ 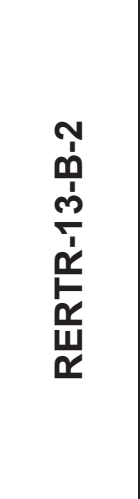 } & B-1 & Blank & $3.30 E+14$ & -- & -- & -- & -- & -- & -- \\
\hline & B-2 & Blank & $3.33 E+14$ & -- & -- & -- & -- & -- & -- \\
\hline & B-3 & Blank & 3.36E+14 & -- & -- & -- & -- & -- & -- \\
\hline & B-4 & Blank & $3.37 E+14$ & -- & -- & -- & -- & -- & -- \\
\hline & B-5 & PCP0G4 & $2.97 E+14$ & 217.29 & -- & -- & -- & -- & -- \\
\hline & B-6 & PAPOFO & $2.85 E+14$ & 301.01 & -- & -- & -- & -- & -- \\
\hline & $B-7$ & PZP0E8 & $2.86 E+14$ & 312.85 & -- & -- & -- & -- & -- \\
\hline & B-8 & PCP0E7 & $2.93 E+14$ & 325.52 & -- & -- & -- & -- & -- \\
\hline \multirow{8}{*}{ 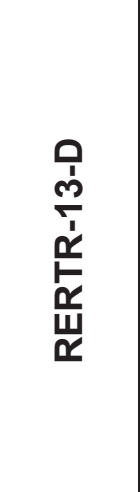 } & D-1 & RCR3F1 & $3.06 \mathrm{E}+14$ & -- & -- & 3259.68 & 103.49 & -- & -- \\
\hline & D-2 & RCR7F5 & $3.15 E+14$ & -- & -- & 5951.20 & 188.95 & -- & -- \\
\hline & D-3 & RZR7F7 & $3.23 E+14$ & -- & -- & 6129.81 & 194.62 & -- & -- \\
\hline & D-4 & RZR3F3 & $3.18 \mathrm{E}+14$ & -- & -- & 3434.14 & 109.03 & -- & -- \\
\hline & D-5 & PBP0F9 & $1.93 E+14$ & 483.11 & -- & -- & -- & -- & -- \\
\hline & D-6 & PAP0G2 & $2.06 E+14$ & 189.67 & -- & -- & -- & -- & -- \\
\hline & D-7 & PZPOE9 & $2.09 E+14$ & 205.32 & -- & -- & -- & -- & -- \\
\hline & D-8 & PCP0D6 & $2.16 E+14$ & 222.42 & -- & -- & -- & -- & -- \\
\hline
\end{tabular}


Table 8: Cycle 151A MOC1 As-Run HGR and Depletion Results for RERTR-13-1 in B-10 Scaled to As-Run Cycle Average East Source Power of 19.7 MW, 15 EFPD 5 .

\begin{tabular}{|c|c|c|c|c|c|c|c|c|c|}
\hline \multirow[b]{2}{*}{$\begin{array}{c}\text { Capsule } \\
\text { ID }\end{array}$} & \multirow[b]{2}{*}{$\begin{array}{c}\text { Plate } \\
\text { Position }\end{array}$} & \multirow[b]{2}{*}{$\begin{array}{l}\text { Plate } \\
\text { ID }\end{array}$} & \multirow[b]{2}{*}{$\begin{array}{c}\text { BA/Fuel } \\
\text { Neutron } \\
\text { Flux } \\
\text { (n/cm²-sec }\end{array}$} & \multicolumn{2}{|c|}{ Burnable Absorber } & \multicolumn{4}{|c|}{ Fuel } \\
\hline & & & & $\begin{array}{l}\text { Power } \\
\text { Density } \\
\text { (W/cc) } \\
\end{array}$ & \begin{tabular}{|c|}
$\%$ \\
Depletion \\
B-10 \\
$(\%)$ \\
\end{tabular} & $\begin{array}{c}\text { Fission } \\
\text { Power } \\
\text { Density } \\
\text { (W/cc) }\end{array}$ & $\begin{array}{c}\text { Surface } \\
\text { Heat } \\
\text { Flux } \\
\left(\mathbf{W} / \mathrm{cm}^{2}\right)\end{array}$ & $\begin{array}{c}\text { Fission } \\
\text { Density } \\
\text { (fissions/cc) }\end{array}$ & \begin{tabular}{|c|}
$\%$ \\
Depletio \\
$\mathbf{n}$ \\
U-235 \\
$(\%)$ \\
\end{tabular} \\
\hline \multirow{8}{*}{ 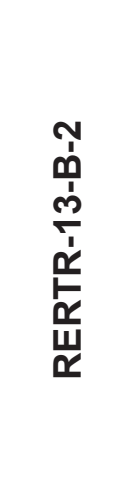 } & B-1 & Blank & $3.08 \mathrm{E}+14$ & -- & -- & -- & -- & -- & -- \\
\hline & B-2 & Blank & $3.11 \mathrm{E}+14$ & -- & -- & -- & -- & -- & -- \\
\hline & B-3 & Blank & $3.13 E+14$ & -- & -- & -- & -- & -- & -- \\
\hline & B-4 & Blank & $3.12 \mathrm{E}+14$ & -- & -- & -- & -- & -- & -- \\
\hline & B-5 & PCP0G4 & $2.92 \mathrm{E}+14$ & 139.34 & $38.87 \%$ & -- & -- & -- & -- \\
\hline & B-6 & PAPOFO & $2.81 \mathrm{E}+14$ & 209.10 & $35.30 \%$ & -- & -- & -- & -- \\
\hline & B-7 & PZP0E8 & $2.81 \mathrm{E}+14$ & 210.96 & $35.44 \%$ & -- & -- & -- & -- \\
\hline & B-8 & PCP0E7 & $2.87 E+14$ & 212.82 & $36.84 \%$ & -- & -- & -- & -- \\
\hline \multirow{8}{*}{ 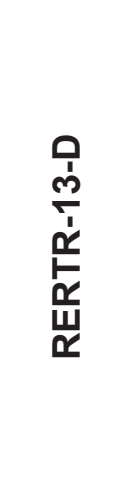 } & D-1 & RCR3F1 & $2.75 E+14$ & -- & $22.74 \%$ & 2667.08 & 84.68 & $1.46 \mathrm{E}+20$ & $4.42 \%$ \\
\hline & D-2 & RCR7F5 & $2.76 E+14$ & -- & $15.86 \%$ & 4696.51 & 149.11 & $2.66 \mathrm{E}+20$ & $3.02 \%$ \\
\hline & D-3 & RZR7F7 & $2.77 \mathrm{E}+14$ & -- & $16.28 \%$ & 4634.00 & 147.13 & $2.74 \mathrm{E}+20$ & $3.02 \%$ \\
\hline & D-4 & RZR3F3 & $2.79 E+14$ & -- & $23.85 \%$ & 2674.05 & 84.90 & $1.54 \mathrm{E}+20$ & $4.63 \%$ \\
\hline & D-5 & PBP0F9 & $1.94 \mathrm{E}+14$ & 403.53 & $20.71 \%$ & -- & -- & -- & -- \\
\hline & D-6 & PAP0G2 & $2.05 E+14$ & 150.19 & $23.87 \%$ & -- & -- & -- & -- \\
\hline & D-7 & PZPOE9 & $2.08 \mathrm{E}+14$ & 157.12 & $24.86 \%$ & -- & -- & -- & -- \\
\hline & D-8 & PCP0D6 & $2.14 \mathrm{E}+14$ & 164.81 & $26.83 \%$ & -- & -- & -- & -- \\
\hline
\end{tabular}


Table 9: Cycle 151A MOC2 As-Run HGR and Depletion Results for RERTR-13-1 in B-10 Scaled to As-Run Cycle Average East Source Power of 19.7 MW, 34 EFPD 5 .

\begin{tabular}{|c|c|c|c|c|c|c|c|c|c|}
\hline \multirow[b]{2}{*}{$\begin{array}{c}\text { Capsule } \\
\text { ID }\end{array}$} & \multirow[b]{2}{*}{$\begin{array}{c}\text { Plate } \\
\text { Position }\end{array}$} & \multirow[b]{2}{*}{$\begin{array}{c}\text { Plate } \\
\text { ID }\end{array}$} & \multirow[b]{2}{*}{$\begin{array}{c}\text { BA/Fuel } \\
\text { Neutron } \\
\text { Flux } \\
\text { (n/cm }{ }^{2}-\mathrm{sec}\end{array}$} & \multicolumn{2}{|c|}{ Burnable Absorber } & \multicolumn{4}{|c|}{ Fuel } \\
\hline & & & & $\begin{array}{l}\text { Power } \\
\text { Density } \\
\text { (W/cc) } \\
\end{array}$ & \begin{tabular}{|c|}
$\%$ \\
Depletion \\
B-10 \\
$(\%)$ \\
\end{tabular} & $\begin{array}{c}\text { Fission } \\
\text { Power } \\
\text { Density } \\
\text { (W/cc) }\end{array}$ & $\begin{array}{c}\text { Surface } \\
\text { Heat } \\
\text { Flux } \\
\left(\mathrm{W} / \mathrm{cm}^{2}\right)\end{array}$ & $\begin{array}{c}\text { Fission } \\
\text { Density } \\
\text { (fissions/cc) }\end{array}$ & \begin{tabular}{|c}
$\%$ \\
Depletion \\
U-235 \\
$(\%)$ \\
\end{tabular} \\
\hline \multirow{8}{*}{ 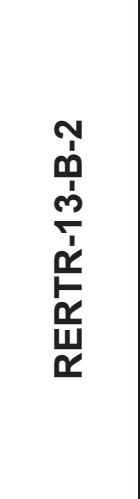 } & $B-1$ & Blank & $3.01 \mathrm{E}+14$ & -- & -- & -- & -- & -- & -- \\
\hline & B-2 & Blank & $3.01 \mathrm{E}+14$ & -- & -- & -- & -- & -- & -- \\
\hline & $B-3$ & Blank & $3.03 E+14$ & -- & -- & -- & -- & -- & -- \\
\hline & B-4 & Blank & $3.05 E+14$ & -- & -- & -- & -- & -- & -- \\
\hline & B-5 & PCP0G4 & $2.93 E+14$ & 81.76 & $67.97 \%$ & -- & -- & -- & -- \\
\hline & B-6 & PAPOF0 & $2.85 E+14$ & 132.35 & $64.16 \%$ & -- & -- & -- & -- \\
\hline & $B-7$ & PZP0E8 & $2.88 E+14$ & 136.73 & $63.91 \%$ & -- & -- & -- & -- \\
\hline & B-8 & PCP0E7 & $2.90 \mathrm{E}+14$ & 133.31 & $65.30 \%$ & -- & -- & -- & -- \\
\hline \multirow{8}{*}{ 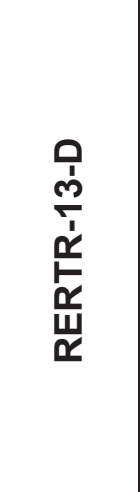 } & D-1 & RCR3F1 & $2.75 E+14$ & -- & $41.63 \%$ & 2635.23 & 83.67 & $2.98 \mathrm{E}+20$ & $8.98 \%$ \\
\hline & D-2 & RCR7F5 & $2.79 E+14$ & -- & $29.60 \%$ & 4832.46 & 153.43 & $5.33 E+20$ & $6.00 \%$ \\
\hline & D-3 & RZR7F7 & $2.78 E+14$ & -- & $29.80 \%$ & 4794.48 & 152.22 & $5.38 \mathrm{E}+20$ & $6.00 \%$ \\
\hline & D-4 & RZR3F3 & $2.77 E+14$ & -- & $42.53 \%$ & 2668.98 & 84.74 & $3.06 E+20$ & $9.21 \%$ \\
\hline & D-5 & PBP0F9 & $1.99 \mathrm{E}+14$ & 329.92 & $41.90 \%$ & -- & -- & -- & -- \\
\hline & D-6 & PAP0G2 & $2.07 E+14$ & 117.92 & $46.89 \%$ & -- & -- & -- & -- \\
\hline & D-7 & PZPOE9 & $2.14 \mathrm{E}+14$ & 123.17 & $48.20 \%$ & -- & -- & -- & -- \\
\hline & D-8 & PCP0D6 & $2.19 E+14$ & 123.75 & $51.00 \%$ & -- & -- & -- & -- \\
\hline
\end{tabular}


Table 10: Cycle 151A EOC As-Run HGR and Depletion Results for RERTR-13-1 in B-10 Scaled to As-Run Cycle Average East Source Power of 19.7 MW, 56.1 EFPD ${ }^{5}$.

\begin{tabular}{|c|c|c|c|c|c|c|c|c|c|}
\hline \multirow[b]{2}{*}{$\begin{array}{c}\text { Capsule } \\
\text { ID }\end{array}$} & \multirow[b]{2}{*}{$\begin{array}{c}\text { Plate } \\
\text { Position }\end{array}$} & \multirow[b]{2}{*}{$\begin{array}{c}\text { Plate } \\
\text { ID }\end{array}$} & \multirow[b]{2}{*}{$\begin{array}{c}\text { BA/Fuel } \\
\text { Neutron } \\
\text { Flux } \\
\left(\mathrm{n} / \mathrm{cm}^{2}-\mathrm{sec}\right) \\
\end{array}$} & \multicolumn{2}{|c|}{ Burnable Absorber } & \multicolumn{4}{|c|}{ Fuel } \\
\hline & & & & $\begin{array}{l}\text { Power } \\
\text { Density } \\
\text { (W/cc) }\end{array}$ & \begin{tabular}{|c|}
$\%$ \\
Depletion \\
B-10 \\
$(\%)$ \\
\end{tabular} & $\begin{array}{l}\text { Fission } \\
\text { Power } \\
\text { Density } \\
\text { (W/cc) } \\
\end{array}$ & $\begin{array}{c}\text { Surface } \\
\text { Heat } \\
\text { Flux } \\
\left(\mathrm{W} / \mathrm{cm}^{2}\right)\end{array}$ & $\begin{array}{c}\text { Fission } \\
\text { Density } \\
\text { (fissions/cc) }\end{array}$ & $\begin{array}{c}\% \\
\text { Depletion } \\
\text { U-235 } \\
(\%) \\
\end{array}$ \\
\hline \multirow{8}{*}{ 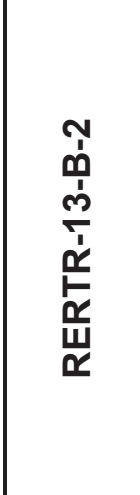 } & B-1 & Blank & $3.02 E+14$ & -- & -- & -- & -- & -- & -- \\
\hline & B-2 & Blank & $3.01 \mathrm{E}+14$ & -- & -- & -- & -- & -- & -- \\
\hline & B-3 & Blank & $3.04 \mathrm{E}+14$ & -- & -- & -- & -- & -- & -- \\
\hline & B-4 & Blank & $3.06 \mathrm{E}+14$ & -- & -- & -- & -- & -- & -- \\
\hline & B-5 & PCP0G4 & $3.03 E+14$ & 43.22 & $85.87 \%$ & -- & -- & -- & -- \\
\hline & B-6 & PAPOFO & $2.99 \mathrm{E}+14$ & 71.76 & $83.53 \%$ & -- & -- & -- & -- \\
\hline & B-7 & PZP0E8 & $3.02 E+14$ & 74.72 & $83.38 \%$ & -- & -- & -- & -- \\
\hline & B-8 & PCP0E7 & $3.03 \mathrm{E}+14$ & 71.51 & $84.17 \%$ & -- & -- & -- & -- \\
\hline \multirow{8}{*}{ 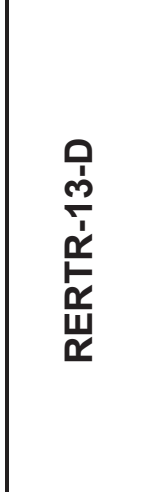 } & D-1 & RCR3F1 & $2.77 E+14$ & -- & $58.44 \%$ & 2608.55 & 82.82 & $4.74 \mathrm{E}+20$ & $14.23 \%$ \\
\hline & D-2 & RCR7F5 & $2.81 \mathrm{E}+14$ & -- & $43.61 \%$ & 4849.79 & 153.98 & $8.56 \mathrm{E}+20$ & $9.59 \%$ \\
\hline & D-3 & RZR7F7 & $2.81 \mathrm{E}+14$ & -- & $43.67 \%$ & 4796.15 & 152.28 & $8.58 E+20$ & $9.59 \%$ \\
\hline & D-4 & RZR3F3 & $2.80 \mathrm{E}+14$ & -- & $59.29 \%$ & 2627.94 & 83.44 & $4.84 \mathrm{E}+20$ & $14.55 \%$ \\
\hline & D-5 & PBP0F9 & $2.10 \mathrm{E}+14$ & 257.73 & $61.31 \%$ & -- & -- & -- & -- \\
\hline & D-6 & PAP0G2 & $2.18 \mathrm{E}+14$ & 85.87 & $66.83 \%$ & -- & -- & -- & -- \\
\hline & D-7 & PZP0E9 & $2.24 \mathrm{E}+14$ & 87.49 & $68.30 \%$ & -- & -- & -- & -- \\
\hline & D-8 & PCP0D6 & $2.28 \mathrm{E}+14$ & 84.15 & $70.9 \%$ & -- & -- & -- & -- \\
\hline
\end{tabular}


Table 11: Cycle 151B BOC As-Run HGR and Depletion Results for RERTR-13-2 in B-10 Scaled to As-Run Cycle Average East Source Power of 19.9 MW, 0EFPD ${ }^{6}$.

\begin{tabular}{|c|c|c|c|c|c|c|c|c|c|}
\hline \multirow[b]{2}{*}{$\begin{array}{c}\text { Capsule } \\
\text { ID }\end{array}$} & \multirow[b]{2}{*}{$\begin{array}{c}\text { Plate } \\
\text { Position } \\
\end{array}$} & \multirow[b]{2}{*}{$\begin{array}{l}\text { Plate } \\
\text { ID }\end{array}$} & \multirow[b]{2}{*}{$\begin{array}{l}\text { BA/Fuel } \\
\text { Neutron } \\
\text { Flux } \\
\left(\mathrm{n} / \mathrm{cm}^{2}-\mathrm{sec}\right)\end{array}$} & \multicolumn{2}{|c|}{ Burnable Absorber } & \multicolumn{4}{|c|}{ Fuel } \\
\hline & & & & $\begin{array}{c}\text { Power } \\
\text { Density } \\
\text { (W/cc) }\end{array}$ & $\begin{array}{c}\% \\
\text { Depletion } \\
\text { B-10 } \\
(\%) \\
\end{array}$ & \begin{tabular}{|c|} 
Fission \\
Power \\
Density \\
(W/cc) \\
\end{tabular} & $\begin{array}{c}\text { Surface } \\
\text { Heat } \\
\text { Flux } \\
\left(\mathrm{W} / \mathrm{cm}^{2}\right) \\
\end{array}$ & $\begin{array}{c}\text { Fission } \\
\text { Density } \\
\text { (fissions/cc) }\end{array}$ & $\begin{array}{c}\% \\
\text { Depletion } \\
\text { U-235 } \\
(\%) \\
\end{array}$ \\
\hline \multirow{8}{*}{ 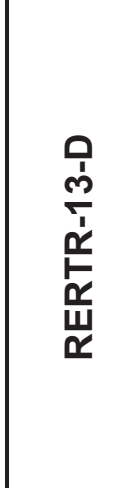 } & D-1 & RCR3F1 & $2.87 E+14$ & -- & $58.44 \%$ & 2760.67 & 87.65 & $4.74 \mathrm{E}+20$ & $14.23 \%$ \\
\hline & D-2 & RCR7F5 & $2.93 E+14$ & -- & $43.61 \%$ & 5112.12 & 162.31 & $8.56 \mathrm{E}+20$ & $9.59 \%$ \\
\hline & D-3 & RZR7F7 & $2.95 E+14$ & -- & $43.67 \%$ & 5117.77 & 162.49 & $8.58 \mathrm{E}+20$ & $9.59 \%$ \\
\hline & D-4 & RZR3F3 & $2.92 E+14$ & -- & $59.29 \%$ & 2799.04 & 88.87 & $4.84 \mathrm{E}+20$ & $14.55 \%$ \\
\hline & D-5 & PBP0F9 & $2.18 \mathrm{E}+14$ & 271.51 & $61.31 \%$ & -- & -- & -- & -- \\
\hline & D-6 & PAP0G2 & $2.29 E+14$ & 90.08 & $66.83 \%$ & -- & -- & -- & -- \\
\hline & D-7 & PZPOE9 & $2.32 \mathrm{E}+14$ & 91.91 & $68.30 \%$ & -- & -- & -- & -- \\
\hline & D-8 & PCP0D6 & $2.36 E+14$ & 87.93 & $70.90 \%$ & -- & -- & -- & -- \\
\hline
\end{tabular}

Table 12: Cycle 151B MOC1 As-Run HGR and Depletion Results for RERTR-13-2 in B-10 Scaled to As-Run Cycle Average East Source Power of 19.9 MW, 23 EFPD ${ }^{6}$.

\begin{tabular}{|c|c|c|c|c|c|c|c|c|c|}
\hline \multirow[b]{2}{*}{\begin{tabular}{|c} 
Capsule \\
ID
\end{tabular}} & \multirow[b]{2}{*}{$\begin{array}{c}\text { Plate } \\
\text { Position }\end{array}$} & \multirow[b]{2}{*}{$\begin{array}{c}\text { Plate } \\
\text { ID }\end{array}$} & \multirow[b]{2}{*}{$\begin{array}{c}\text { BA/Fuel } \\
\text { Neutron } \\
\text { Flux } \\
\left(\mathrm{n} / \mathrm{cm}^{2}-\mathrm{sec}\right) \\
\end{array}$} & \multicolumn{2}{|c|}{ Burnable Absorber } & \multicolumn{4}{|c|}{ Fuel } \\
\hline & & & & $\begin{array}{l}\text { Power } \\
\text { Density } \\
\text { (W/cc) } \\
\end{array}$ & \begin{tabular}{|c}
$\%$ \\
Depletion \\
B-10 \\
$(\%)$ \\
\end{tabular} & $\begin{array}{c}\text { Fission } \\
\text { Power } \\
\text { Density } \\
\text { (W/cc) }\end{array}$ & $\begin{array}{c}\text { Surface } \\
\text { Heat } \\
\text { Flux } \\
\left(\mathrm{W} / \mathrm{cm}^{2}\right) \\
\end{array}$ & $\begin{array}{c}\text { Fission } \\
\text { Density } \\
\text { (fissions/cc) }\end{array}$ & \begin{tabular}{|c|}
$\%$ \\
Depletion \\
U-235 \\
$(\%)$ \\
\end{tabular} \\
\hline \multirow{8}{*}{ 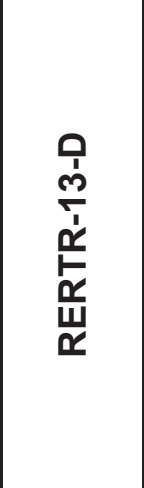 } & D-1 & RCR3F1 & $2.78 \mathrm{E}+14$ & -- & $71.72 \%$ & 2504.30 & 79.51 & $6.63 E+20$ & $19.83 \%$ \\
\hline & D-2 & RCR7F5 & $2.84 \mathrm{E}+14$ & -- & $56.13 \%$ & 4801.33 & 152.44 & $1.21 \mathrm{E}+21$ & $13.44 \%$ \\
\hline & D-3 & RZR7F7 & $2.89 E+14$ & -- & $56.18 \%$ & 4904.23 & 155.71 & $1.21 \mathrm{E}+21$ & $13.50 \%$ \\
\hline & D-4 & RZR3F3 & $2.89 E+14$ & -- & $72.50 \%$ & 2646.83 & 84.04 & $6.76 \mathrm{E}+20$ & $20.19 \%$ \\
\hline & D-5 & PBP0F9 & $2.25 E+14$ & 178.82 & $76.86 \%$ & -- & -- & -- & -- \\
\hline & D-6 & PAP0G2 & 2.37E+14 & 56.68 & $81.47 \%$ & -- & -- & -- & -- \\
\hline & D-7 & PZP0E9 & $2.40 E+14$ & 56.70 & $82.70 \%$ & -- & -- & -- & -- \\
\hline & D-8 & PCP0D6 & $2.44 \mathrm{E}+14$ & 52.79 & $84.51 \%$ & -- & -- & -- & -- \\
\hline
\end{tabular}


Table 13: Cycle 151B MOC2 As-Run HGR and Depletion Results for RERTR-13-2 in B-10

Scaled to As-Run Cycle Average East Source Power of 19.9 MW, 39EFPD ${ }^{6}$.

\begin{tabular}{|c|c|c|c|c|c|c|c|c|c|}
\hline \multirow[b]{2}{*}{$\begin{array}{c}\text { Capsule } \\
\text { ID }\end{array}$} & \multirow[b]{2}{*}{$\begin{array}{c}\text { Plate } \\
\text { Position }\end{array}$} & \multirow[b]{2}{*}{$\begin{array}{c}\text { Plate } \\
\text { ID }\end{array}$} & \multirow[b]{2}{*}{$\begin{array}{c}\text { BA/Fuel } \\
\text { Neutron } \\
\text { Flux } \\
\text { (n/cm }{ }^{2}-\mathrm{sec}\end{array}$} & \multicolumn{2}{|c|}{ Burnable Absorber } & \multicolumn{4}{|c|}{ Fuel } \\
\hline & & & & $\begin{array}{l}\text { Power } \\
\text { Density } \\
\text { (W/cc) } \\
\end{array}$ & \begin{tabular}{|c|}
$\%$ \\
Depletion \\
B-10 \\
$(\%)$ \\
\end{tabular} & $\begin{array}{c}\text { Fission } \\
\text { Power } \\
\text { Density } \\
\text { (W/cc) }\end{array}$ & $\begin{array}{c}\text { Surface } \\
\text { Heat } \\
\text { Flux } \\
\left(\mathrm{W} / \mathrm{cm}^{2}\right) \\
\end{array}$ & $\begin{array}{c}\text { Fission } \\
\text { Density } \\
\text { (fissions/cc) } \\
\end{array}$ & \begin{tabular}{|c|}
$\%$ \\
Depletion \\
U-235 \\
$(\%)$ \\
\end{tabular} \\
\hline \multirow{8}{*}{ 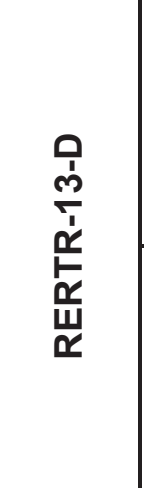 } & D-1 & RCR3F1 & $3.39 E+14$ & -- & $78.16 \%$ & 3522.17 & 111.83 & $7.83 E+20$ & $23.37 \%$ \\
\hline & D-2 & RCR7F5 & $3.42 E+14$ & -- & $63.01 \%$ & 6570.18 & 208.60 & $1.44 \mathrm{E}+21$ & $15.99 \%$ \\
\hline & D-3 & RZR7F7 & $3.45 E+14$ & -- & $63.22 \%$ & 6607.63 & 209.79 & $1.44 \mathrm{E}+21$ & $16.11 \%$ \\
\hline & D-4 & RZR3F3 & $3.42 E+14$ & -- & $79.09 \%$ & 3522.29 & 111.83 & $8.02 E+20$ & $23.94 \%$ \\
\hline & D-5 & PBP0F9 & $2.77 E+14$ & 186.85 & $84.32 \%$ & -- & -- & -- & -- \\
\hline & D-6 & PAP0G2 & $2.89 \mathrm{E}+14$ & 55.17 & $88.03 \%$ & -- & -- & -- & -- \\
\hline & D-7 & PZP0E9 & $2.91 \mathrm{E}+14$ & 52.95 & $89.05 \%$ & -- & -- & -- & -- \\
\hline & D-8 & PCP0D6 & $2.94 \mathrm{E}+14$ & 48.24 & $90.32 \%$ & -- & -- & -- & -- \\
\hline
\end{tabular}

Table 14: Cycle 151B EOC As-Run HGR and Depletion Results for RERTR-13-2 in B-10 Scaled to As-Run Cycle Average East Source Power of 19.9 MW, 51.3 EFPD ${ }^{6}$.

\begin{tabular}{|c|c|c|c|c|c|c|c|c|c|}
\hline \multirow[b]{2}{*}{$\begin{array}{l}\text { Capsule } \\
\text { ID }\end{array}$} & \multirow[b]{2}{*}{\begin{tabular}{|c} 
Plate \\
Position
\end{tabular}} & \multirow[b]{2}{*}{$\begin{array}{l}\text { Plate } \\
\text { ID }\end{array}$} & \multirow[b]{2}{*}{$\begin{array}{l}\text { BA/Fuel } \\
\text { Neutron } \\
\text { Flux } \\
\left(\mathrm{n} / \mathrm{cm}^{2}-\mathrm{sec}\right)\end{array}$} & \multicolumn{2}{|c|}{ Burnable Absorber } & \multicolumn{4}{|c|}{ Fuel } \\
\hline & & & & $\begin{array}{l}\text { Power } \\
\text { Density } \\
\text { (W/cc) }\end{array}$ & $\begin{array}{c}\% \\
\text { Depletion } \\
\text { B-10 } \\
(\%) \\
\end{array}$ & $\begin{array}{c}\text { Fission } \\
\text { Power } \\
\text { Density } \\
\text { (W/cc) }\end{array}$ & $\begin{array}{c}\text { Surface } \\
\text { Heat } \\
\text { Flux } \\
\left(\mathrm{W} / \mathrm{cm}^{2}\right)\end{array}$ & $\begin{array}{c}\text { Fission } \\
\text { Density } \\
\text { (fissions/cc) }\end{array}$ & \begin{tabular}{|c|}
$\%$ \\
Depletio \\
$\mathbf{n}$ \\
U-235 \\
$(\%)$ \\
\end{tabular} \\
\hline \multirow{8}{*}{ 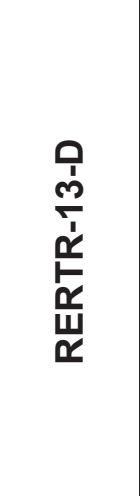 } & D-1 & RCR3F1 & $3.30 E+14$ & -- & $83.70 \%$ & 3297.79 & 104.70 & $9.13 E+20$ & $27.17 \%$ \\
\hline & D-2 & RCR7F5 & $3.33 E+14$ & -- & $69.32 \%$ & 6173.80 & 196.02 & $1.68 \mathrm{E}+21$ & $18.66 \%$ \\
\hline & D-3 & RZR7F7 & $3.36 \mathrm{E}+14$ & -- & $69.52 \%$ & 6231.69 & 197.86 & $1.69 \mathrm{E}+21$ & $18.77 \%$ \\
\hline & D-4 & RZR3F3 & $3.34 \mathrm{E}+14$ & -- & $84.44 \%$ & 3310.72 & 105.12 & $9.32 E+20$ & $27.74 \%$ \\
\hline & D-5 & PBP0F9 & $2.85 \mathrm{E}+14$ & 127.16 & $90.15 \%$ & -- & -- & -- & -- \\
\hline & D-6 & PAP0G2 & $2.96 \mathrm{E}+14$ & 37.03 & $92.79 \%$ & -- & -- & -- & -- \\
\hline & D-7 & PZP0E9 & $2.96 \mathrm{E}+14$ & 35.08 & $93.46 \%$ & -- & -- & -- & -- \\
\hline & D-8 & PCP0D6 & $2.98 \mathrm{E}+14$ & 31.84 & $94.26 \%$ & -- & -- & -- & -- \\
\hline
\end{tabular}




\subsection{Gradients}

MCNP-calculated power gradients have not yet been performed for this experiment.

\section{HYDRAULIC TESTING}

A fully assembled irradiation test vehicle (with simulated fuel plates) was used for testing. The test vehicle was fabricated such that the orifice plates could be easily changed. The hydraulic resistance of the RERTR Large B-Position irradiation test vehicle with various orifice plate sizes were calculated, the results are shown in Table 15.

Table 15: Loss Coefficients for the RERTR Irradiation Test Vehicle Components ${ }^{7}$

\begin{tabular}{ccc}
\hline $\begin{array}{c}\text { Orifice Dia. } \\
(\mathrm{mm})\end{array}$ & $\begin{array}{c}\mathrm{K} / \mathrm{A}^{2} \\
\left(1 / \mathrm{m}^{4}\right)\end{array}$ & $\begin{array}{c}\text { ATR Coolant } \\
\text { Flow Rate } \\
\left(\mathrm{cm}^{3} / \mathrm{sec}\right)\end{array}$ \\
\hline 10 & $5.3041 \times 10^{8}$ & 1252 \\
\hline 9 & $8.2181 \times 10^{8}$ & 1046 \\
\hline 8 & $1.6961 \times 10^{9}$ & 757 \\
\hline 7.32 & $2.9022 \times 10^{9}$ & 588 \\
\hline 7 & $3.0058 \times 10^{9}$ & 579 \\
\hline 6 & $4.0784 \times 10^{9}$ & 500 \\
\hline 5 & $101743 \times 10^{10}$ & 298 \\
\hline Bypass & $2.7958 \times 10^{8}$ & -- \\
Vehicle & $1.4161 \times 10^{8}$ & 2727 \\
\hline
\end{tabular}

Based on the results from the hydraulic testing, the orifice was removed leaving the capsule in the "Vehicle" configuration to provide an ATR coolant flow rate through the capsules of $2727 \mathrm{~cm}^{3} / \mathrm{sec}^{8}$

\section{AS-RUN THERMAL ANALYSIS}

The thermal as-run analysis was performed using the as-built geometry, MCNP-calculated surface heat flux $\left(\mathrm{W} / \mathrm{cm}^{2}\right)$ and nominal coolant channel flow rate. ABAQUS ${ }^{9}$ was used to calculate the coolant channel temperatures and plate surface temperatures.

The heat transfer correlation used to calculate these temperatures was calculated from the Colburn equation (equation 5-50c from Reference 10):

$$
N u=\frac{h D}{k}=0.023 \operatorname{Re}^{0.8} \operatorname{Pr}^{0.3}
$$

Where $\mathrm{Nu}$ is the Nusselt number, $\mathrm{h}$ is the heat transfer coefficient, $\mathrm{D}$ is the hydraulic diameter, $\mathrm{k}$ is the thermal conductivity, Re is the Reynolds number and Pr is the Prandlt number.

The thermal analysis was performed using the beginning of life L2ARs shown in Section 0. 


\subsection{Coolant Channel Temperature}

The coolant temperature was analyzed at the five flow channels in the capsule ${ }^{11}$ (see Figure 9). For each interval, the coolant temperature was plotted as a function of location along the test assembly with 0.0 in. being at the top of the assembly. These plots are shown in Figure 10 through Figure 17.

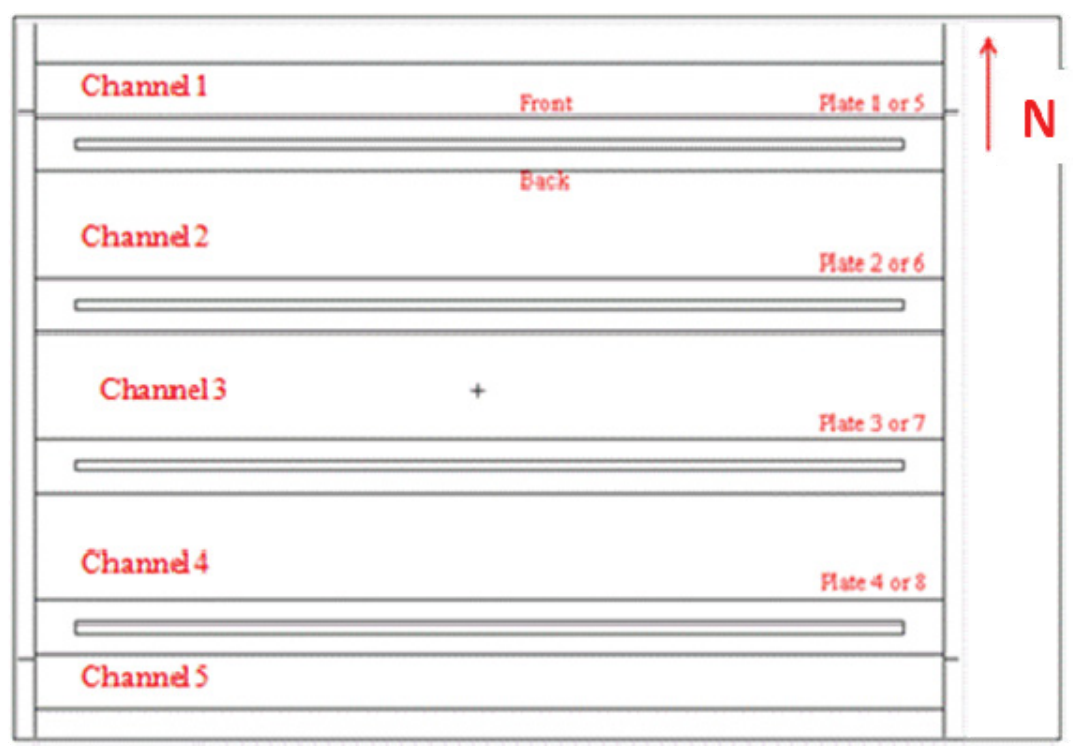

Figure 9: RERTR-13 capsule cross section with the plate IDs (not shown) and plate 1 facing north.

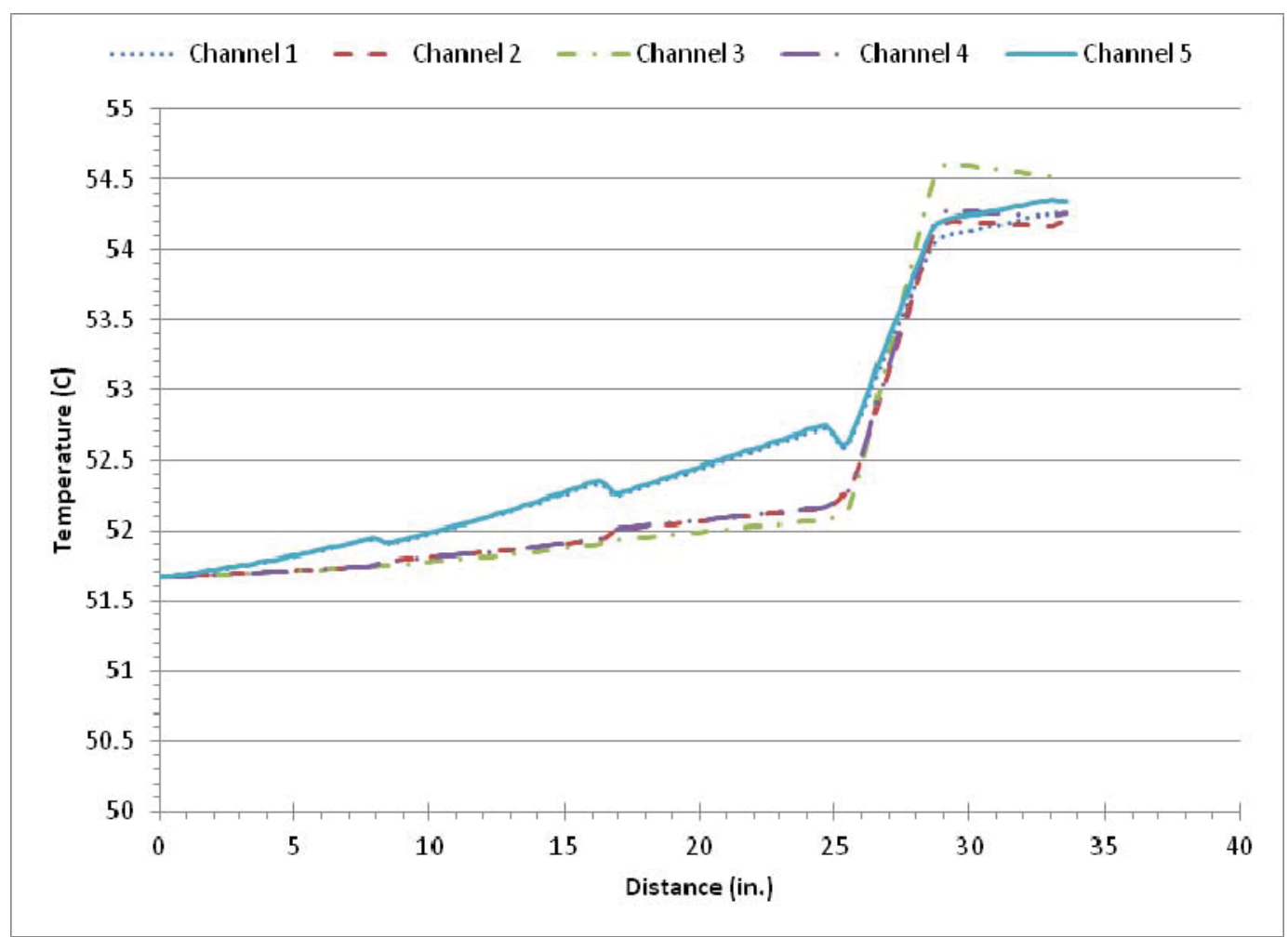

Figure 10: Coolant channel temperatures as a function of location along the RERTR-13 test assembly at BOC 151A (0.0 EFPD). 


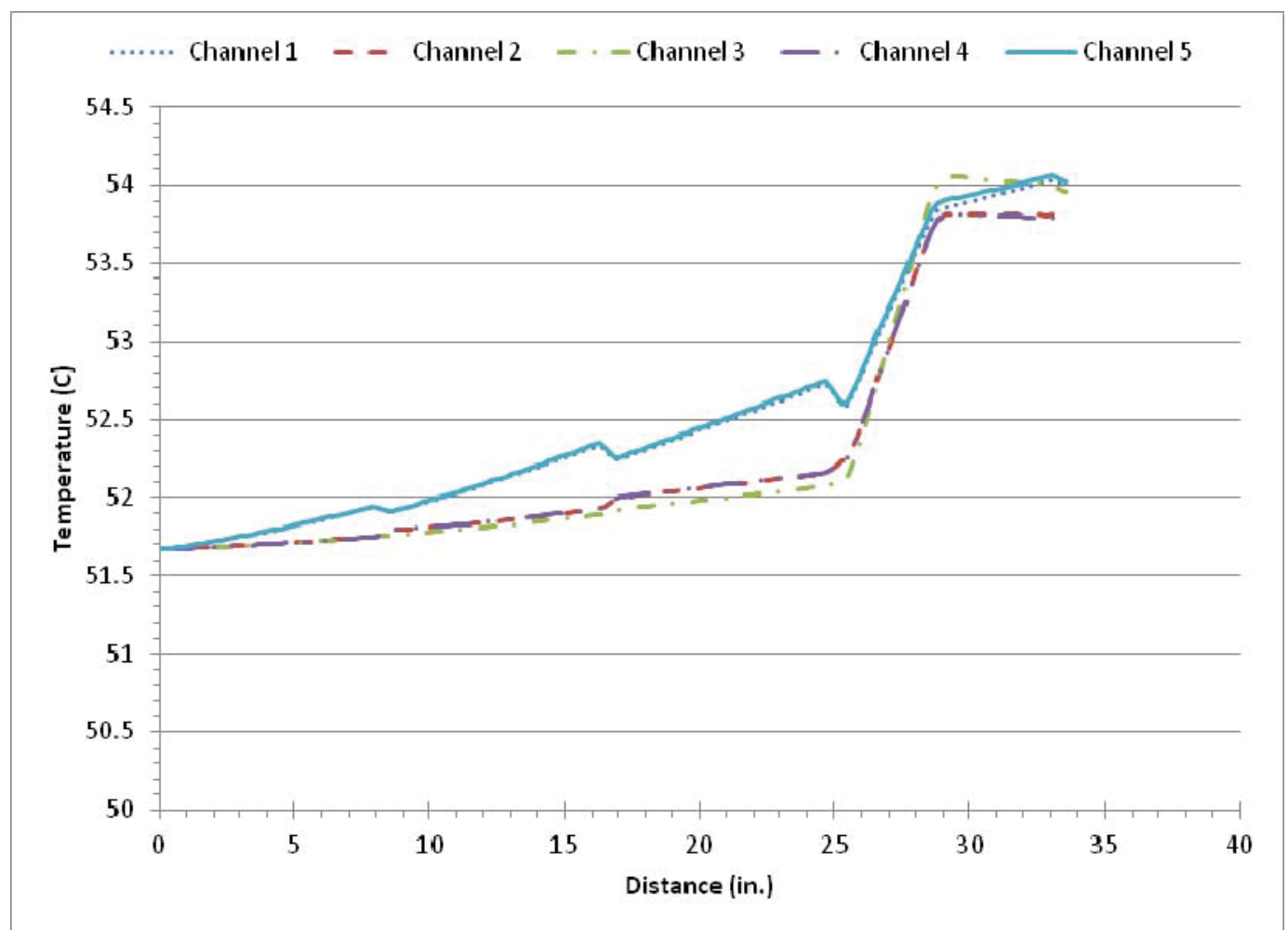

Figure 11: Coolant channel temperature as a function of location along the RERTR-13 test assembly at MOC1 151A (15.0 EFPD).

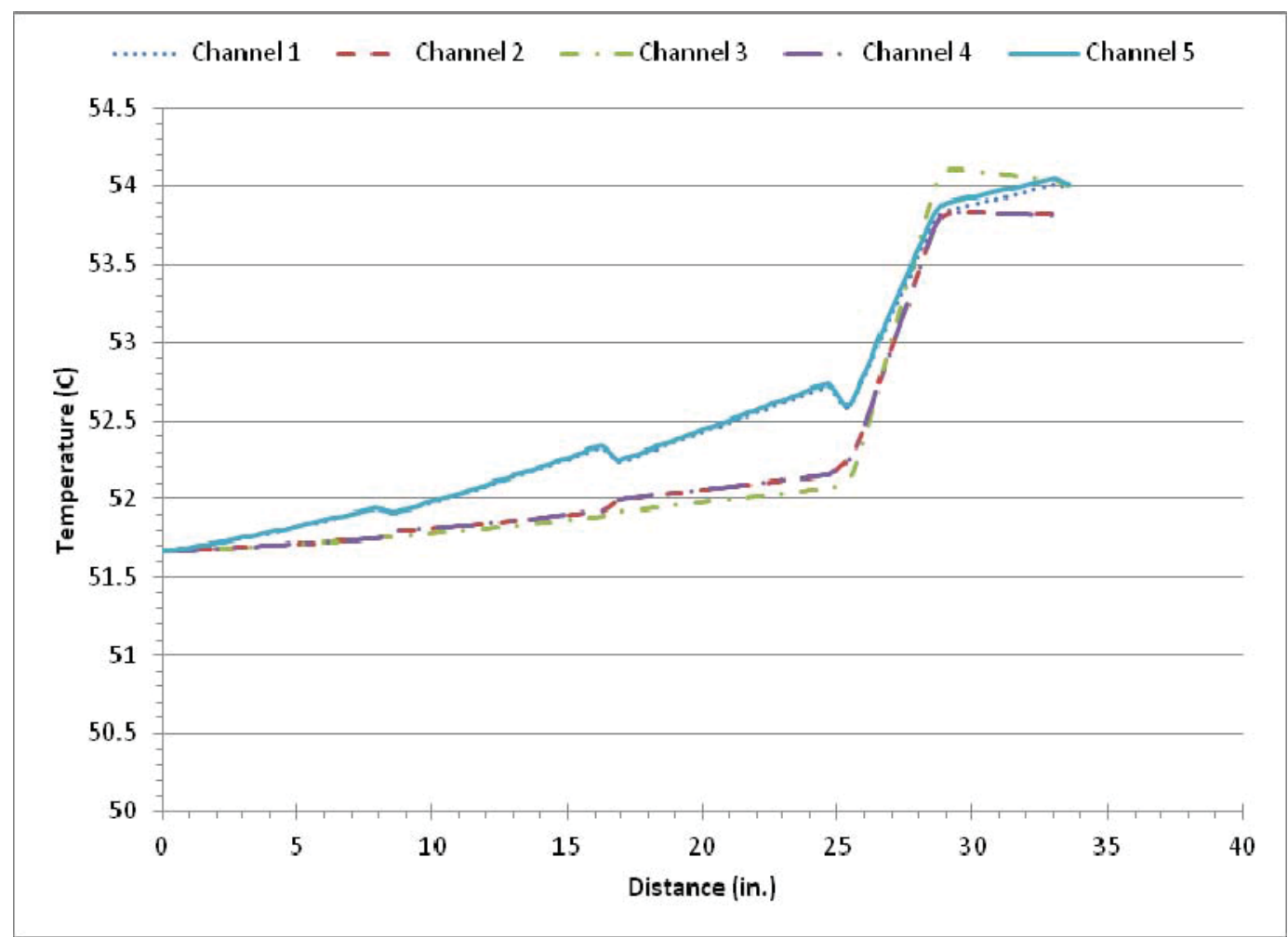

Figure 12: Coolant channel temperature as a function of location along the RERTR-13 test assembly at MOC2 151A (34.0 EFPD). 


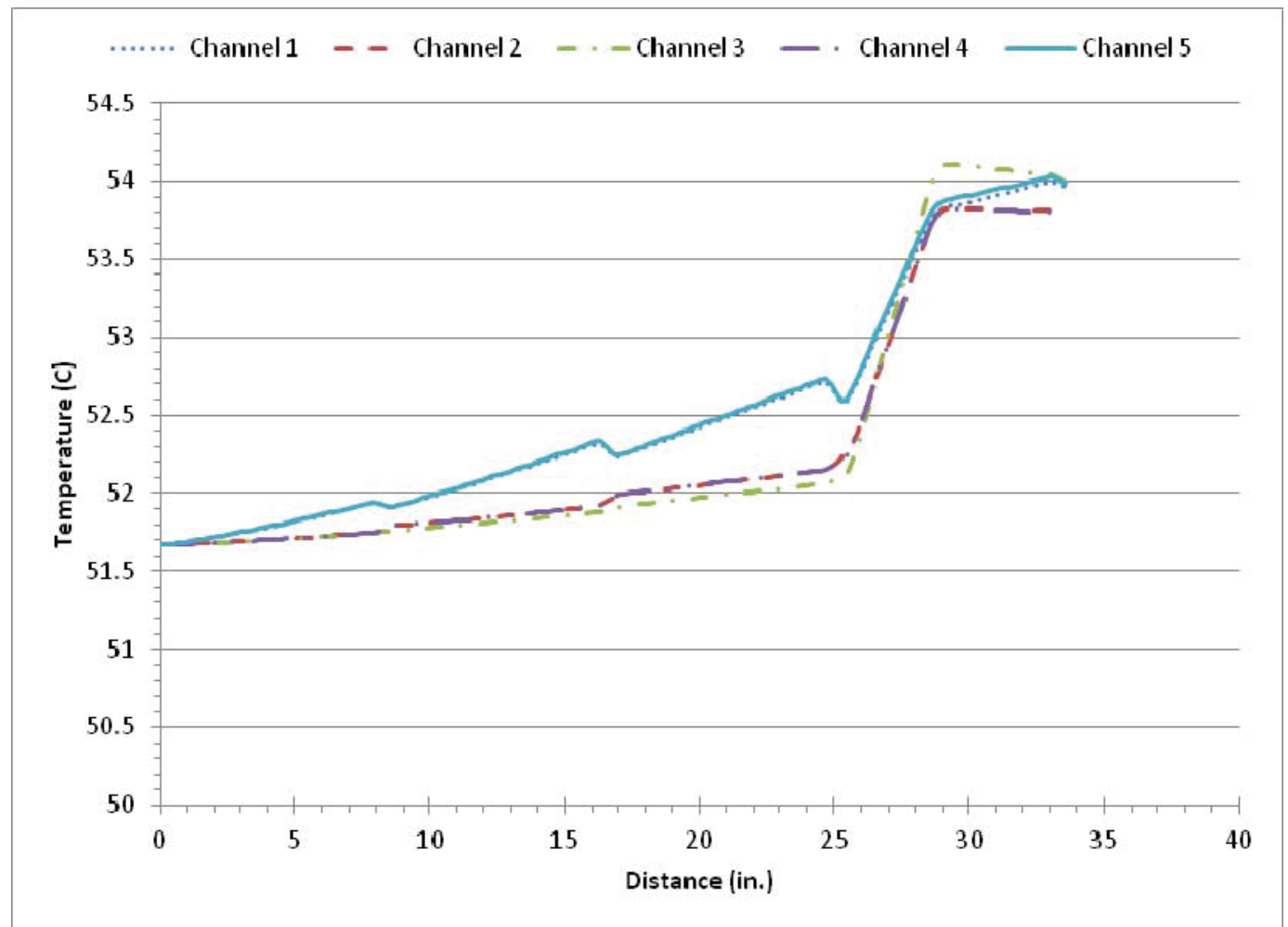

Figure 13: Coolant channel temperature as a function of location along the RERTR-13 test assembly at EOC 151A (56.1 EFPD).

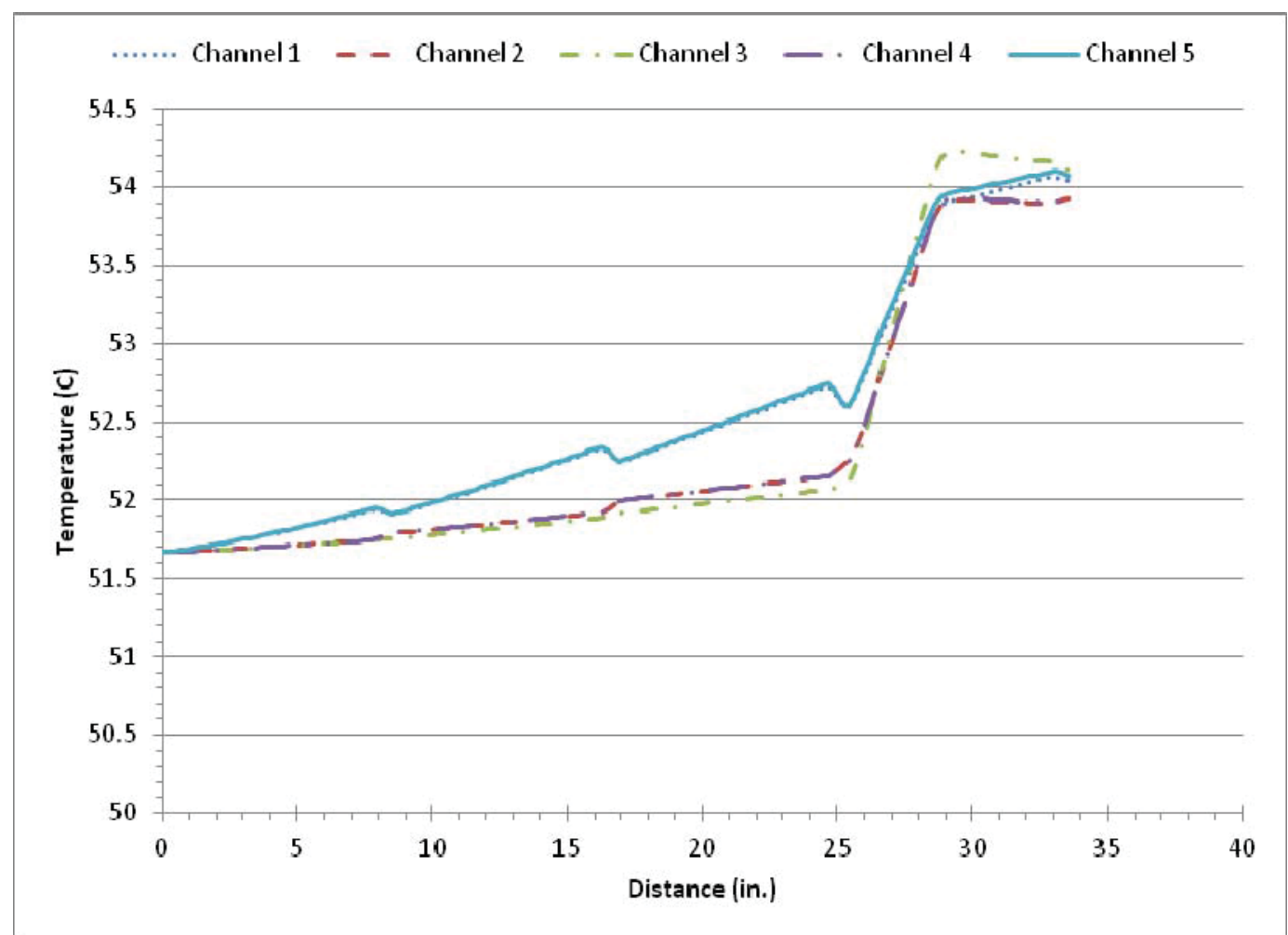

Figure 14: Coolant channel temperature as a function of location along the RERTR-13 test assembly at BOC 151B (0.0 EFPD). 


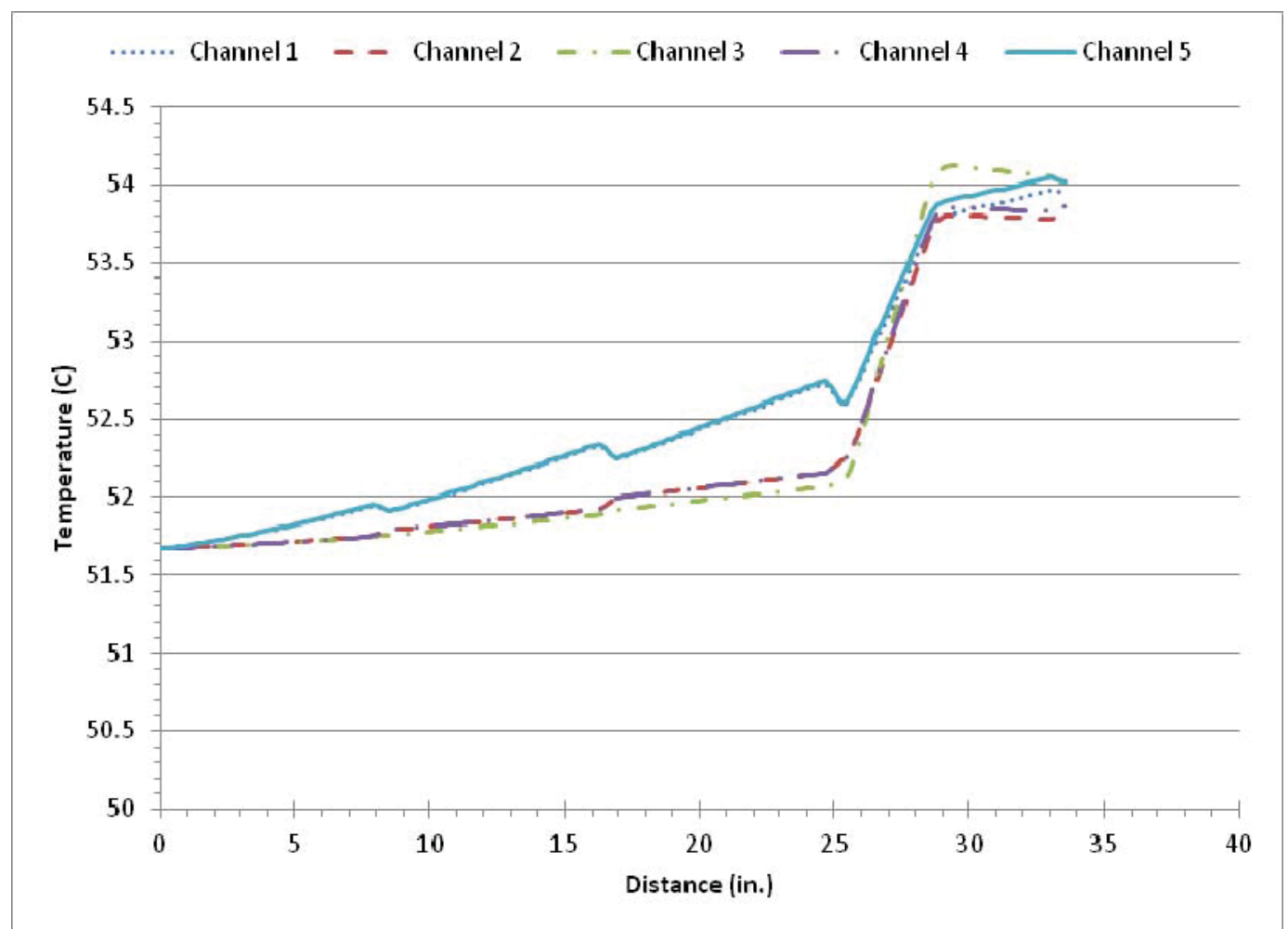

Figure 15: Coolant channel temperature as a function of location along the RERTR-13 test assembly at MOC1 151B (23.0 EFPD).

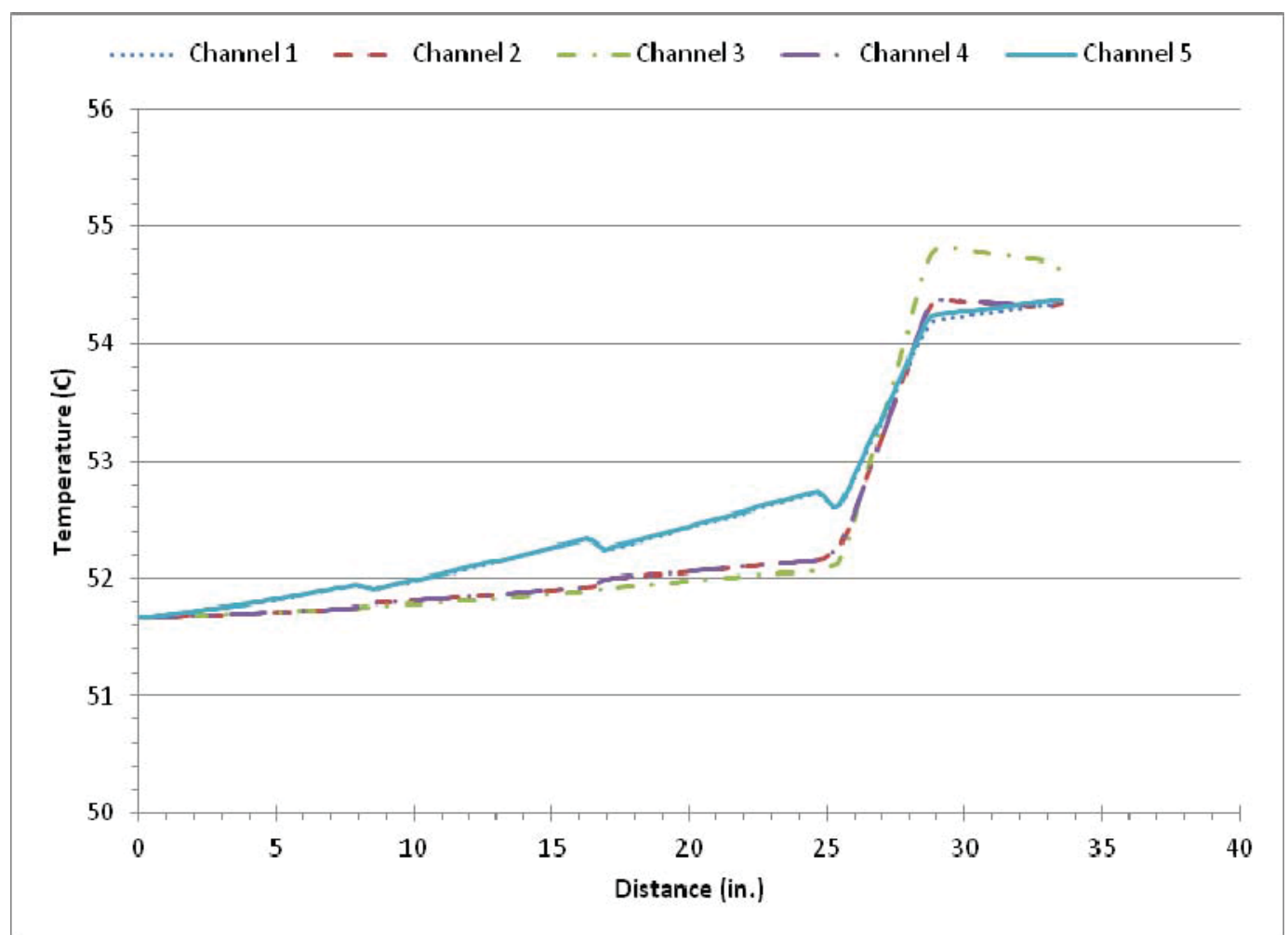

Figure 16: Coolant channel temperature as a function of location along the RERTR-13 test assembly at MOC2 151B (39.0 EFPD). 


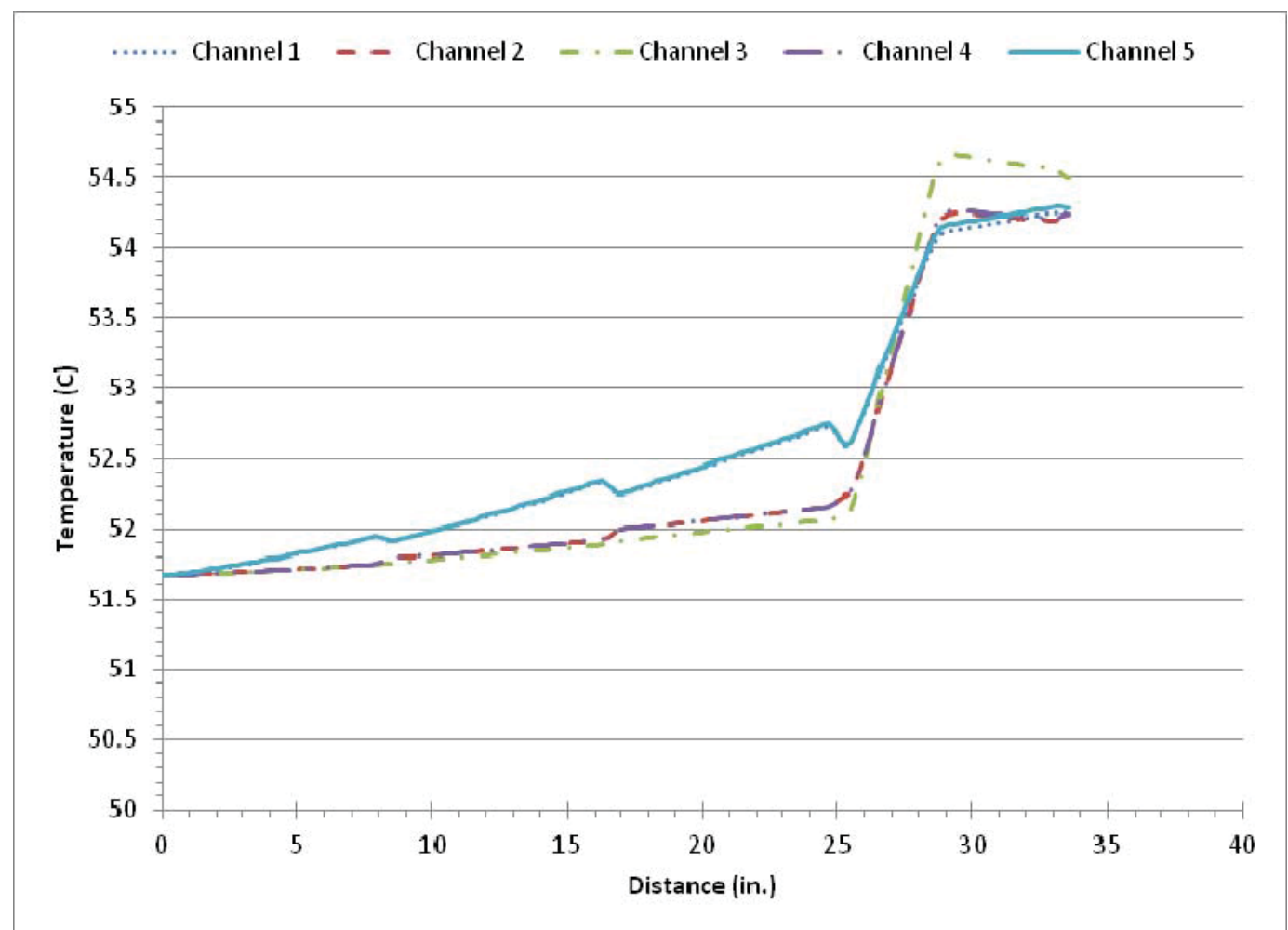

Figure 17: Coolant channel temperature as a function of location along the RERTR-13 test assembly at EOC 151B (51.3 EFPD). 


\subsection{Plate Surface Temperature}

The minimum, maximum and average plate surface temperatures over the fuel zone on each side of the plate are shown in Table 16 through Table 31, where the plate ID is facing north ${ }^{11}$ (see Figure 9).

Table 16: As-run minimum, maximum and average plate surface temperatures over fuel zone on the north side of the plate for capsules irradiated in Cycle 151A, BOC (0.0 EFPD)

\begin{tabular}{|c|c|c|c|c|}
\hline $\begin{array}{c}\text { Plate } \\
\text { Location }\end{array}$ & Plate ID & $\begin{array}{c}\text { Minimum } \\
\text { Temperature } \\
(\mathrm{C})\end{array}$ & $\begin{array}{c}\text { Maximum } \\
\text { Temperature } \\
(\mathrm{C})\end{array}$ & $\begin{array}{c}\text { Average } \\
\text { Temperature } \\
(\mathrm{C})\end{array}$ \\
\hline B-5 & PCP0G4 & 52.34 & 53.02 & 52.68 \\
\hline B-6 & PAP0F0 & 52.20 & 52.75 & 52.47 \\
\hline B-7 & PZP0E8 & 52.20 & 52.75 & 52.47 \\
\hline B-8 & PCP0E7 & 52.34 & 52.96 & 52.62 \\
\hline \hline D-1 & RCR3F1 & 58.27 & 76.20 & 69.25 \\
\hline D-2 & RCR7F5 & 61.44 & 89.68 & 78.98 \\
\hline D-3 & RZR7F7 & 61.66 & 90.79 & 79.70 \\
\hline D-4 & RZR3F3 & 58.36 & 76.24 & 69.28 \\
\hline D-5 & PBP0F9 & 54.47 & 55.03 & 54.84 \\
\hline D-6 & PAP0G2 & 54.35 & 54.70 & 54.50 \\
\hline D-7 & PZP0E9 & 54.45 & 54.89 & 54.68 \\
\hline D-8 & PCP0D6 & 54.39 & 54.83 & 54.58 \\
\hline
\end{tabular}

Table 17: As-run minimum, maximum and average plate surface temperatures over fuel zone on the south side of the plate for capsules irradiated in Cycle 151A, BOC (0.0 EFPD)

\begin{tabular}{|c|c|c|c|c|}
\hline $\begin{array}{c}\text { Plate } \\
\text { Location }\end{array}$ & Plate ID & $\begin{array}{c}\text { Minimum } \\
\text { Temperature } \\
(\mathrm{C})\end{array}$ & $\begin{array}{c}\text { Maximum } \\
\text { Temperature } \\
(\mathrm{C})\end{array}$ & $\begin{array}{c}\text { Average } \\
\text { Temperature } \\
(\mathrm{C})\end{array}$ \\
\hline B-5 & PCP0G4 & 52.27 & 52.89 & 52.50 \\
\hline B-6 & PAP0F0 & 52.19 & 52.74 & 52.46 \\
\hline B-7 & PZP0E8 & 52.21 & 52.76 & 52.49 \\
\hline B-8 & PCP0E7 & 52.41 & 53.10 & 52.80 \\
\hline \hline D-1 & RCR3F1 & 58.10 & 75.15 & 68.50 \\
\hline D-2 & RCR7F5 & 61.42 & 89.86 & 79.01 \\
\hline D-3 & RZR7F7 & 61.69 & 90.65 & 79.68 \\
\hline D-4 & RZR3F3 & 58.55 & 77.36 & 70.07 \\
\hline D-5 & PBP0F9 & 54.44 & 54.94 & 54.76 \\
\hline D-6 & PAP0G2 & 54.41 & 54.86 & 54.65 \\
\hline D-7 & PZP0E9 & 54.40 & 54.75 & 54.56 \\
\hline D-8 & PCP0D6 & 54.42 & 54.93 & 54.65 \\
\hline
\end{tabular}


Table 18: As-run minimum, maximum and average plate surface temperatures over fuel zone on the north side of the plate for capsules irradiated in Cycle 151A, MOC1 (15.0 EFPD)

\begin{tabular}{|c|c|c|c|c|}
\hline $\begin{array}{c}\text { Plate } \\
\text { Location }\end{array}$ & Plate ID & $\begin{array}{c}\text { Minimum } \\
\text { Temperature } \\
(\mathrm{C})\end{array}$ & $\begin{array}{c}\text { Maximum } \\
\text { Temperature } \\
\text { (C) }\end{array}$ & $\begin{array}{c}\text { Average } \\
\text { Temperature } \\
\text { (C) }\end{array}$ \\
\hline B-5 & PCP0G4 & 52.29 & 52.96 & 52.59 \\
\hline B-6 & PAP0F0 & 52.15 & 52.69 & 52.38 \\
\hline B-7 & PZP0E8 & 52.14 & 52.68 & 52.36 \\
\hline B-8 & PCP0E7 & 52.28 & 52.89 & 52.50 \\
\hline \hline D-1 & RCR3F1 & 57.38 & 72.29 & 66.47 \\
\hline D-2 & RCR7F5 & 59.71 & 82.76 & 73.95 \\
\hline D-3 & RZR7F7 & 59.60 & 82.52 & 73.71 \\
\hline D-4 & RZR3F3 & 57.24 & 71.39 & 65.85 \\
\hline D-5 & PBP0F9 & 54.18 & 54.74 & 54.52 \\
\hline D-6 & PAP0G2 & 53.98 & 54.34 & 54.10 \\
\hline D-7 & PZP0E9 & 54.02 & 54.38 & 54.19 \\
\hline D-8 & PCP0D6 & 54.02 & 54.48 & 54.16 \\
\hline
\end{tabular}

Table 19: As-run minimum, maximum and average plate surface temperatures over fuel zone on the south side of the plate for capsules irradiated in Cycle 151A, MOC1 (15.0 EFPD)

\begin{tabular}{|c|c|c|c|c|}
\hline $\begin{array}{c}\text { Plate } \\
\text { Location }\end{array}$ & Plate ID & $\begin{array}{c}\text { Minimum } \\
\text { Temperature } \\
(\mathrm{C})\end{array}$ & $\begin{array}{c}\text { Maximum } \\
\text { Temperature } \\
(\mathrm{C})\end{array}$ & $\begin{array}{c}\text { Average } \\
\text { Temperature } \\
(\mathrm{C})\end{array}$ \\
\hline B-5 & PCP0G4 & 52.22 & 52.83 & 52.42 \\
\hline B-6 & PAP0F0 & 52.14 & 52.68 & 52.36 \\
\hline B-7 & PZP0E8 & 52.15 & 52.69 & 52.38 \\
\hline B-8 & PCP0E7 & 52.34 & 53.02 & 52.68 \\
\hline \hline D-1 & RCR3F1 & 57.22 & 71.35 & 65.81 \\
\hline D-2 & RCR7F5 & 59.69 & 82.86 & 73.96 \\
\hline D-3 & RZR7F7 & 59.63 & 82.41 & 73.71 \\
\hline D-4 & RZR3F3 & 57.40 & 72.37 & 66.52 \\
\hline D-5 & PBP0F9 & 54.14 & 54.62 & 54.39 \\
\hline D-6 & PAP0G2 & 54.01 & 54.38 & 54.18 \\
\hline D-7 & PZP0E9 & 53.98 & 54.34 & 54.10 \\
\hline D-8 & PCP0D6 & 54.07 & 54.60 & 54.29 \\
\hline
\end{tabular}


Table 20: As-run minimum, maximum and average plate surface temperatures over fuel zone on the north side of the plate for capsules irradiated in Cycle 151A, MOC2 (34.0 EFPD)

\begin{tabular}{|c|c|c|c|c|}
\hline $\begin{array}{c}\text { Plate } \\
\text { Location }\end{array}$ & Plate ID & $\begin{array}{c}\text { Minimum } \\
\text { Temperature } \\
(\mathrm{C})\end{array}$ & $\begin{array}{c}\text { Maximum } \\
\text { Temperature } \\
(\mathrm{C})\end{array}$ & $\begin{array}{c}\text { Average } \\
\text { Temperature } \\
(\mathrm{C})\end{array}$ \\
\hline B-5 & PCP0G4 & 52.26 & 52.92 & 52.53 \\
\hline B-6 & PAP0F0 & 52.10 & 52.64 & 52.30 \\
\hline B-7 & PZP0E8 & 52.10 & 52.63 & 52.29 \\
\hline B-8 & PCP0E7 & 52.22 & 52.83 & 52.41 \\
\hline \hline D-1 & RCR3F1 & 57.33 & 72.08 & 66.32 \\
\hline D-2 & RCR7F5 & 59.89 & 83.51 & 74.50 \\
\hline D-3 & RZR7F7 & 59.82 & 83.42 & 74.36 \\
\hline D-4 & RZR3F3 & 57.23 & 71.37 & 65.83 \\
\hline D-5 & PBP0F9 & 54.14 & 54.69 & 54.43 \\
\hline D-6 & PAP0G2 & 53.96 & 54.34 & 54.09 \\
\hline D-7 & PZP0E9 & 54.03 & 54.39 & 54.19 \\
\hline D-8 & PCP0D6 & 53.98 & 54.46 & 54.13 \\
\hline
\end{tabular}

Table 21: As-run minimum, maximum and average plate surface temperatures over fuel zone on the south side of the plate for capsules irradiated in Cycle 151A, MOC2 (34.0 EFPD)

\begin{tabular}{|c|c|c|c|c|}
\hline $\begin{array}{c}\text { Plate } \\
\text { Location }\end{array}$ & Plate ID & $\begin{array}{c}\text { Minimum } \\
\text { Temperature } \\
(\mathrm{C})\end{array}$ & $\begin{array}{c}\text { Maximum } \\
\text { Temperature } \\
(\mathrm{C})\end{array}$ & $\begin{array}{c}\text { Average } \\
\text { Temperature } \\
(\mathrm{C})\end{array}$ \\
\hline B-5 & PCP0G4 & 52.14 & 52.79 & 52.36 \\
\hline B-6 & PAP0F0 & 52.09 & 52.63 & 52.28 \\
\hline B-7 & PZP0E8 & 52.10 & 52.64 & 52.30 \\
\hline B-8 & PCP0E7 & 52.29 & 52.97 & 52.59 \\
\hline \hline D-1 & RCR3F1 & 57.17 & 71.16 & 65.67 \\
\hline D-2 & RCR7F5 & 59.87 & 83.63 & 74.51 \\
\hline D-3 & RZR7F7 & 59.84 & 83.31 & 74.35 \\
\hline D-4 & RZR3F3 & 57.39 & 72.34 & 66.50 \\
\hline D-5 & PBP0F9 & 54.10 & 54.58 & 54.32 \\
\hline D-6 & PAP0G2 & 54.03 & 54.39 & 54.19 \\
\hline D-7 & PZP0E9 & 53.97 & 54.34 & 54.10 \\
\hline D-8 & PCP0D6 & 54.05 & 54.58 & 54.25 \\
\hline
\end{tabular}


Table 22: As-run minimum, maximum and average plate surface temperatures over fuel zone on the north side of the plate for capsules irradiated in Cycle 151A, EOC (56.1 EFPD)

\begin{tabular}{|c|c|c|c|c|}
\hline $\begin{array}{c}\text { Plate } \\
\text { Location }\end{array}$ & Plate ID & $\begin{array}{c}\text { Minimum } \\
\text { Temperature } \\
(\mathrm{C})\end{array}$ & $\begin{array}{c}\text { Maximum } \\
\text { Temperature } \\
(\mathrm{C})\end{array}$ & $\begin{array}{c}\text { Average } \\
\text { Temperature } \\
(\mathrm{C})\end{array}$ \\
\hline B-5 & PCP0G4 & 52.23 & 52.89 & 52.48 \\
\hline B-6 & PAP0F0 & 52.07 & 52.60 & 52.23 \\
\hline B-7 & PZP0E8 & 52.05 & 52.59 & 52.22 \\
\hline B-8 & PCP0E7 & 52.12 & 52.79 & 52.35 \\
\hline \hline D-1 & RCR3F1 & 57.29 & 71.90 & 66.19 \\
\hline D-2 & RCR7F5 & 59.91 & 83.60 & 74.57 \\
\hline D-3 & RZR7F7 & 59.82 & 83.43 & 74.37 \\
\hline D-4 & RZR3F3 & 57.17 & 71.11 & 65.64 \\
\hline D-5 & PBP0F9 & 54.09 & 54.63 & 54.35 \\
\hline D-6 & PAP0G2 & 53.91 & 54.31 & 54.05 \\
\hline D-7 & PZP0E9 & 54.01 & 54.36 & 54.15 \\
\hline D-8 & PCP0D6 & 53.91 & 54.42 & 54.08 \\
\hline
\end{tabular}

Table 23: As-run minimum, maximum and average plate surface temperatures over fuel zone on the south side of the plate for capsules irradiated in Cycle 151A, EOC (56.1 EFPD)

\begin{tabular}{|c|c|c|c|c|}
\hline $\begin{array}{c}\text { Plate } \\
\text { Location }\end{array}$ & Plate ID & $\begin{array}{c}\text { Minimum } \\
\text { Temperature } \\
(\mathrm{C})\end{array}$ & $\begin{array}{c}\text { Maximum } \\
\text { Temperature } \\
(\mathrm{C})\end{array}$ & $\begin{array}{c}\text { Average } \\
\text { Temperature } \\
(\mathrm{C})\end{array}$ \\
\hline B-5 & PCP0G4 & 52.08 & 52.77 & 52.32 \\
\hline B-6 & PAP0F0 & 52.05 & 52.59 & 52.22 \\
\hline B-7 & PZP0E8 & 52.07 & 52.60 & 52.24 \\
\hline B-8 & PCP0E7 & 52.25 & 52.92 & 52.52 \\
\hline \hline D-1 & RCR3F1 & 57.13 & 70.99 & 65.55 \\
\hline D-2 & RCR7F5 & 59.89 & 83.72 & 74.58 \\
\hline D-3 & RZR7F7 & 59.84 & 83.31 & 74.36 \\
\hline D-4 & RZR3F3 & 57.33 & 72.06 & 66.30 \\
\hline D-5 & PBP0F9 & 54.06 & 54.52 & 54.24 \\
\hline D-6 & PAP0G2 & 54.01 & 54.36 & 54.15 \\
\hline D-7 & PZP0E9 & 53.92 & 54.31 & 54.05 \\
\hline D-8 & PCP0D6 & 54.01 & 54.54 & 54.19 \\
\hline
\end{tabular}


Table 24: As-run minimum, maximum and average plate surface temperatures over fuel zone on the north side of the plate for capsules irradiated in Cycle 151B, BOC (0.0 EFPD)

\begin{tabular}{|c|c|c|c|c|}
\hline $\begin{array}{c}\text { Plate } \\
\text { Location }\end{array}$ & Plate ID & $\begin{array}{c}\text { Minimum } \\
\text { Temperature } \\
(\mathrm{C})\end{array}$ & $\begin{array}{c}\text { Maximum } \\
\text { Temperature } \\
\text { (C) }\end{array}$ & $\begin{array}{c}\text { Average } \\
\text { Temperature } \\
\text { (C) }\end{array}$ \\
\hline \hline D-1 & RCR3F1 & 57.53 & 72.93 & 66.93 \\
\hline D-2 & RCR7F5 & 60.29 & 85.08 & 75.64 \\
\hline D-3 & RZR7F7 & 60.28 & 85.25 & 75.68 \\
\hline D-4 & RZR3F3 & 57.43 & 72.23 & 66.43 \\
\hline D-5 & PBP0F9 & 54.17 & 54.71 & 54.43 \\
\hline D-6 & PAP0G2 & 54.00 & 54.40 & 54.15 \\
\hline D-7 & PZP0E9 & 54.11 & 54.46 & 54.26 \\
\hline D-8 & PCP0D6 & 54.01 & 54.51 & 54.17 \\
\hline
\end{tabular}

Table 25: As-run minimum, maximum and average plate surface temperatures over fuel zone on the south side of the plate for capsules irradiated in Cycle 151B, BOC (0.0 EFPD)

\begin{tabular}{|c|c|c|c|c|}
\hline $\begin{array}{c}\text { Plate } \\
\text { Location }\end{array}$ & Plate ID & $\begin{array}{c}\text { Minimum } \\
\text { Temperature } \\
\text { (C) }\end{array}$ & $\begin{array}{c}\text { Maximum } \\
\text { Temperature } \\
\text { (C) }\end{array}$ & $\begin{array}{c}\text { Average } \\
\text { Temperature } \\
\text { (C) }\end{array}$ \\
\hline \hline D-1 & RCR3F1 & 57.37 & 71.98 & 66.26 \\
\hline D-2 & RCR7F5 & 60.27 & 85.21 & 75.66 \\
\hline D-3 & RZR7F7 & 60.30 & 85.12 & 75.67 \\
\hline D-4 & RZR3F3 & 57.60 & 73.21 & 67.12 \\
\hline D-5 & PBP0F9 & 54.14 & 54.61 & 54.33 \\
\hline D-6 & PAP0G2 & 54.10 & 54.46 & 54.26 \\
\hline D-7 & PZP0E9 & 54.01 & 54.41 & 54.15 \\
\hline D-8 & PCP0D6 & 54.10 & 54.62 & 54.28 \\
\hline
\end{tabular}

Table 26: As-run minimum, maximum and average plate surface temperatures over fuel zone on the north side of the plate for capsules irradiated in Cycle 151B, MOC1 (23.0 EFPD)

\begin{tabular}{|c|c|c|c|c|}
\hline $\begin{array}{c}\text { Plate } \\
\text { Location }\end{array}$ & Plate ID & $\begin{array}{c}\text { Minimum } \\
\text { Temperature } \\
\text { (C) }\end{array}$ & $\begin{array}{c}\text { Maximum } \\
\text { Temperature } \\
\text { (C) }\end{array}$ & $\begin{array}{c}\text { Average } \\
\text { Temperature } \\
\text { (C) }\end{array}$ \\
\hline \hline D-1 & RCR3F1 & 57.15 & 71.21 & 65.71 \\
\hline D-2 & RCR7F5 & 59.86 & 83.33 & 74.38 \\
\hline D-3 & RZR7F7 & 59.98 & 84.03 & 74.81 \\
\hline D-4 & RZR3F3 & 57.21 & 71.25 & 65.74 \\
\hline D-5 & PBP0F9 & 54.02 & 54.57 & 54.25 \\
\hline D-6 & PAP0G2 & 53.87 & 54.30 & 54.02 \\
\hline D-7 & PZP0E9 & 54.01 & 54.37 & 54.14 \\
\hline D-8 & PCP0D6 & 53.90 & 54.43 & 54.07 \\
\hline
\end{tabular}


Table 27: As-run minimum, maximum and average plate surface temperatures over fuel zone on the south side of the plate for capsules irradiated in Cycle 151B, MOC1 (23.0 EFPD)

\begin{tabular}{|c|c|c|c|c|}
\hline $\begin{array}{c}\text { Plate } \\
\text { Location }\end{array}$ & Plate ID & $\begin{array}{c}\text { Minimum } \\
\text { Temperature } \\
\text { (C) }\end{array}$ & $\begin{array}{c}\text { Maximum } \\
\text { Temperature } \\
\text { (C) }\end{array}$ & $\begin{array}{c}\text { Average } \\
\text { Temperature } \\
\text { (C) }\end{array}$ \\
\hline \hline D-1 & RCR3F1 & 56.99 & 70.34 & 65.09 \\
\hline D-2 & RCR7F5 & 59.84 & 83.47 & 74.39 \\
\hline D-3 & RZR7F7 & 60.00 & 83.92 & 74.80 \\
\hline D-4 & RZR3F3 & 57.37 & 72.20 & 66.41 \\
\hline D-5 & PBP0F9 & 54.00 & 54.46 & 54.15 \\
\hline D-6 & PAP0G2 & 54.00 & 54.36 & 54.13 \\
\hline D-7 & PZP0E9 & 53.90 & 54.32 & 54.05 \\
\hline D-8 & PCP0D6 & 54.02 & 54.55 & 54.19 \\
\hline
\end{tabular}

Table 28: As-run minimum, maximum and average plate surface temperatures over fuel zone on the north side of the plate for capsules irradiated in Cycle 151B, MOC2 (39.0 EFPD)

\begin{tabular}{|c|c|c|c|c|}
\hline $\begin{array}{c}\text { Plate } \\
\text { Location }\end{array}$ & Plate ID & $\begin{array}{c}\text { Minimum } \\
\text { Temperature } \\
\text { (C) }\end{array}$ & $\begin{array}{c}\text { Maximum } \\
\text { Temperature } \\
\text { (C) }\end{array}$ & $\begin{array}{c}\text { Average } \\
\text { Temperature } \\
\text { (C) }\end{array}$ \\
\hline \hline D-1 & RCR3F1 & 58.67 & 77.90 & 70.45 \\
\hline D-2 & RCR7F5 & 62.28 & 92.95 & 81.37 \\
\hline D-3 & RZR7F7 & 62.31 & 93.34 & 81.55 \\
\hline D-4 & RZR3F3 & 58.50 & 76.82 & 69.68 \\
\hline D-5 & PBP0F9 & 54.43 & 54.93 & 54.63 \\
\hline D-6 & PAP0G2 & 54.33 & 54.75 & 54.52 \\
\hline D-7 & PZP0E9 & 54.51 & 54.86 & 54.68 \\
\hline D-8 & PCP0D6 & 54.30 & 54.79 & 54.47 \\
\hline
\end{tabular}

Table 29: As-run minimum, maximum and average plate surface temperatures over fuel zone on the south side of the plate for capsules irradiated in Cycle 151B, MOC2 (39.0 EFPD)

\begin{tabular}{|c|c|c|c|c|}
\hline $\begin{array}{c}\text { Plate } \\
\text { Location }\end{array}$ & Plate ID & $\begin{array}{c}\text { Minimum } \\
\text { Temperature } \\
\text { (C) }\end{array}$ & $\begin{array}{c}\text { Maximum } \\
\text { Temperature } \\
\text { (C) }\end{array}$ & $\begin{array}{c}\text { Average } \\
\text { Temperature } \\
(\mathrm{C})\end{array}$ \\
\hline \hline D-1 & RCR3F1 & 58.49 & 76.80 & 69.67 \\
\hline D-2 & RCR7F5 & 62.26 & 93.15 & 81.41 \\
\hline D-3 & RZR7F7 & 62.33 & 93.15 & 81.51 \\
\hline D-4 & RZR3F3 & 58.68 & 77.93 & 70.47 \\
\hline D-5 & PBP0F9 & 54.42 & 54.86 & 54.59 \\
\hline D-6 & PAP0G2 & 54.50 & 54.87 & 54.68 \\
\hline D-7 & PZP0E9 & 54.33 & 54.76 & 54.52 \\
\hline D-8 & PCP0D6 & 54.34 & 54.86 & 54.52 \\
\hline
\end{tabular}


Table 30: As-run minimum, maximum and average plate surface temperatures over fuel zone on the north side of the plate for capsules irradiated in Cycle 151B, EOC (51.3 EFPD)

\begin{tabular}{|c|c|c|c|c|}
\hline $\begin{array}{c}\text { Plate } \\
\text { Location }\end{array}$ & Plate ID & $\begin{array}{c}\text { Minimum } \\
\text { Temperature } \\
\text { (C) }\end{array}$ & $\begin{array}{c}\text { Maximum } \\
\text { Temperature } \\
\text { (C) }\end{array}$ & $\begin{array}{c}\text { Average } \\
\text { Temperature } \\
\text { (C) }\end{array}$ \\
\hline \hline D-1 & RCR3F1 & 58.34 & 76.45 & 69.43 \\
\hline D-2 & RCR7F5 & 61.74 & 90.86 & 79.84 \\
\hline D-3 & RZR7F7 & 61.80 & 91.34 & 80.09 \\
\hline D-4 & RZR3F3 & 58.19 & 75.50 & 68.74 \\
\hline D-5 & PBP0F9 & 54.31 & 54.81 & 54.48 \\
\hline D-6 & PAP0G2 & 54.20 & 54.64 & 54.39 \\
\hline D-7 & PZP0E9 & 54.39 & 54.72 & 54.54 \\
\hline D-8 & PCP0D6 & 54.19 & 54.69 & 54.36 \\
\hline
\end{tabular}

Table 31: As-run minimum, maximum and average plate surface temperatures over fuel zone on the south side of the plate for capsules irradiated in Cycle 151B, EOC (51.3 EFPD)

\begin{tabular}{|c|c|c|c|c|}
\hline $\begin{array}{c}\text { Plate } \\
\text { Location }\end{array}$ & Plate ID & $\begin{array}{c}\text { Minimum } \\
\text { Temperature } \\
\text { (C) }\end{array}$ & $\begin{array}{c}\text { Maximum } \\
\text { Temperature } \\
\text { (C) }\end{array}$ & $\begin{array}{c}\text { Average } \\
\text { Temperature } \\
(\mathrm{C})\end{array}$ \\
\hline \hline D-1 & RCR3F1 & 58.16 & 75.41 & 68.68 \\
\hline D-2 & RCR7F5 & 61.72 & 91.04 & 79.87 \\
\hline D-3 & RZR7F7 & 61.82 & 91.17 & 80.06 \\
\hline D-4 & RZR3F3 & 58.37 & 76.57 & 69.51 \\
\hline D-5 & PBP0F9 & 54.29 & 54.73 & 54.44 \\
\hline D-6 & PAP0G2 & 54.38 & 54.72 & 54.54 \\
\hline D-7 & PZP0E9 & 54.21 & 54.65 & 54.39 \\
\hline D-8 & PCP0D6 & 54.24 & 54.78 & 54.42 \\
\hline
\end{tabular}




\section{REFERENCES}

1. "Experiment Control Plan for the RERTR-13 Irradiation in the ATR," PLN-3512, Revision 4, October 2011.

2. RERTR Project Personnel, "RERTR-13 As-Built Data Package” RERTR-13, Job ID 3826810 , February 2012.

3. RERTR Project Personnel, "RERTR-13 As-Built Data Package (Part one, Common Processes)" RERTR-13, Job ID 3302585, November 2010.

4. RERTR Project Personnel, "RERTR-13 As-Built Data Package (Part two, Plates),"RERTR-13, Job ID 3302587, November 2010.

5. M. A. Lillo, G. S. Chang, "RERTR-13 Cycle 151A As-Run Physics Analysis Results and RERTR-13 Cycle 151B Projected Physics Analysis Results,” ECAR-1794, February 2012.

6. M. A. Lillo, G. S. Chang, "RERTR-13 Cycle 151B As-Run Physics Analysis Results,” ECAR-1984, June 2012.

7. D.M. Wachs, "RERTR-Large-B Position Irradiation Vehicle Flow Test” EDF-8292, July 2007.

8. D. M. Wachs, "Thermal Analysis of the RERTR-9B Irradiation Test," EDF-8083, July 2007.

9. P. E. Murray, "Validation of ABAQUS Standard 6.7-3 Heat Transfer", ECAR-131, January 2008.

10. R. H. Perry, D. W. Green, "Perry's Chemical Engineer's Handbook," $7^{\text {th }}$ Edition, McGraw-Hill, 1997.

11. G. A. Roth, "As-Run Thermal Analysis for the RERTR-13 Experiment Irradiated in the ATR" ECAR-2029, September 2012. 


\section{Appendix A}

\section{Individual Plate Power and Fission Density Plots}




\section{Appendix A \\ Individual Plate Power and Fission Density Plots}

A-1. Capsule B
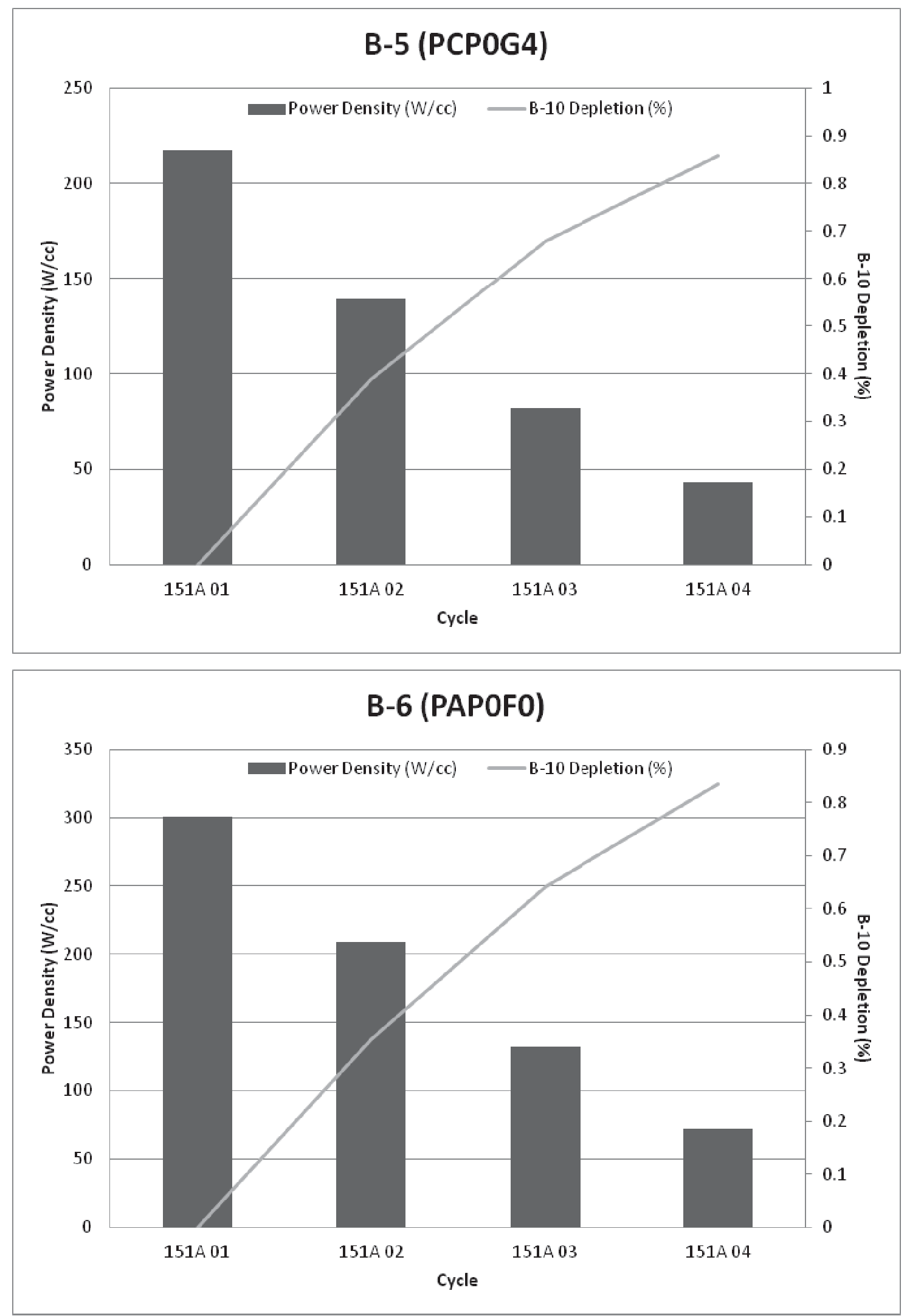

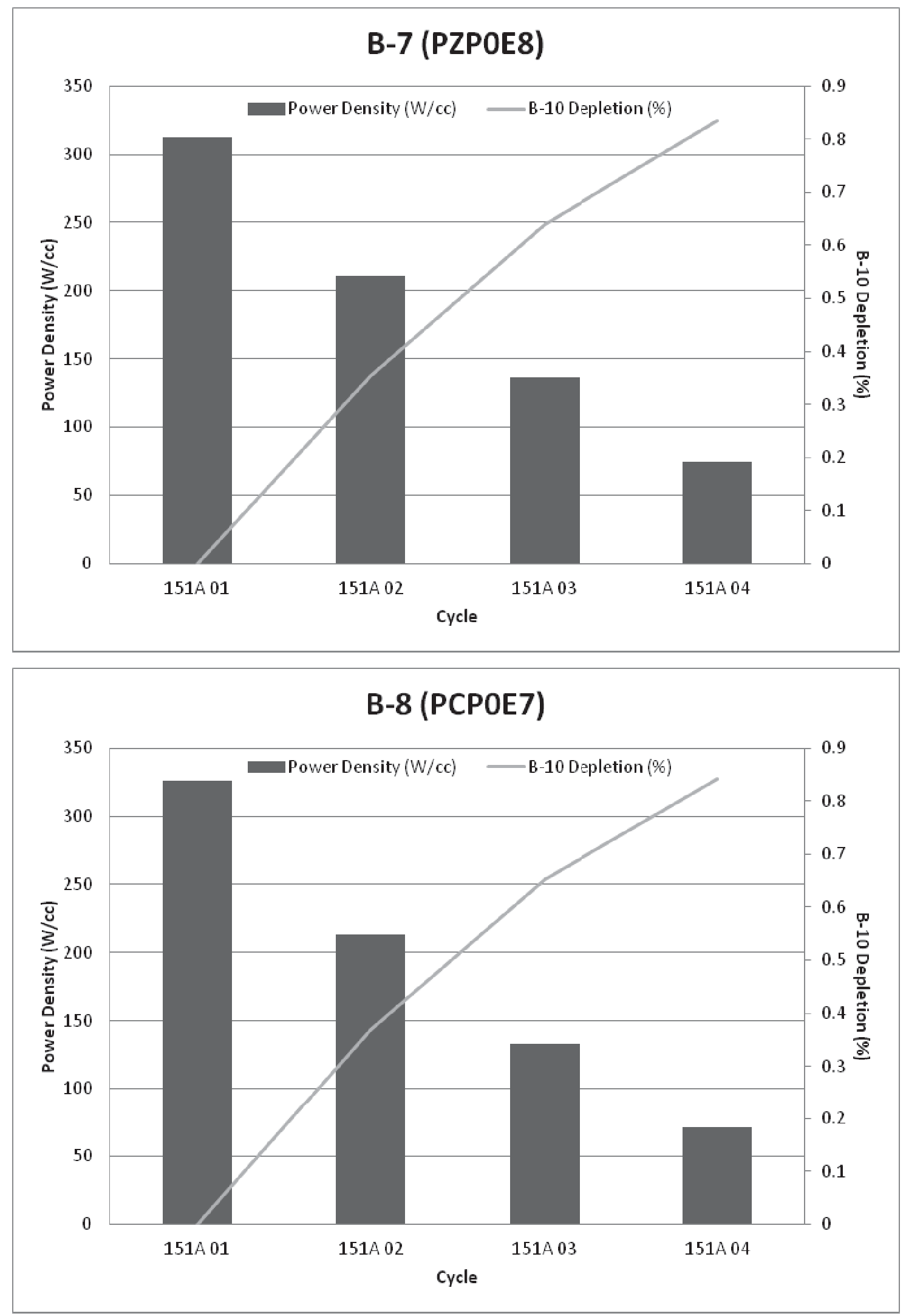


\section{A-2. Capsule D}

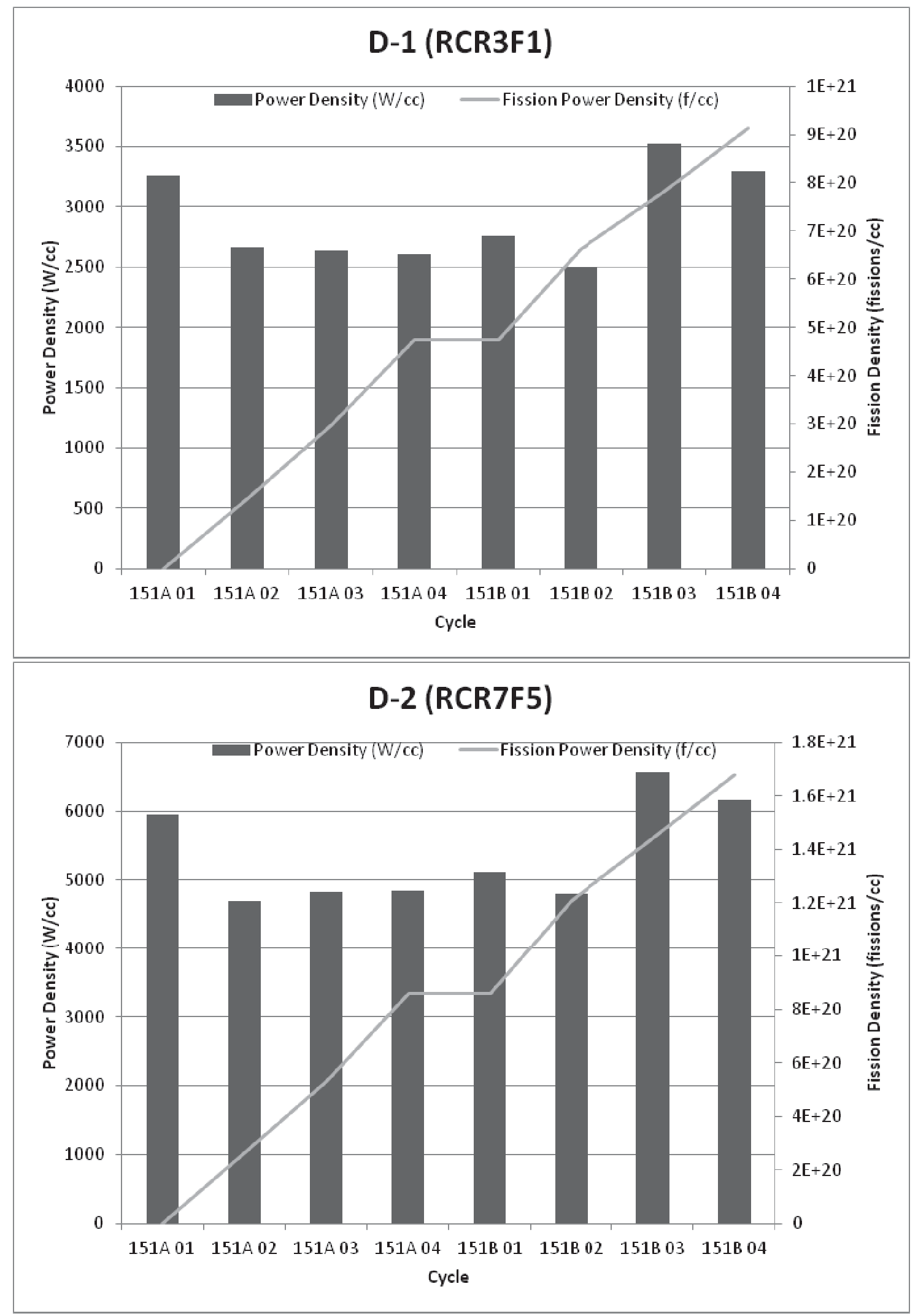



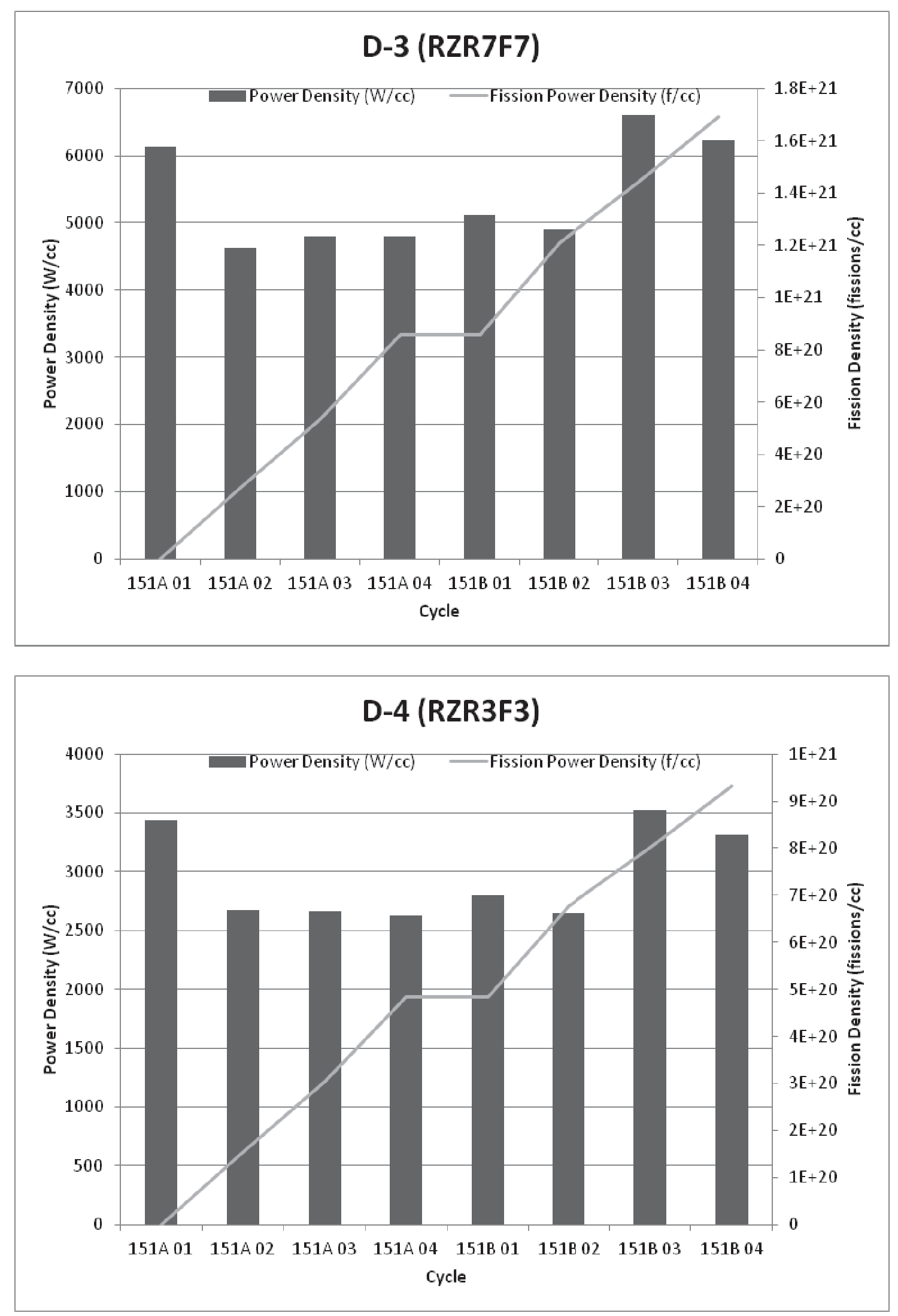

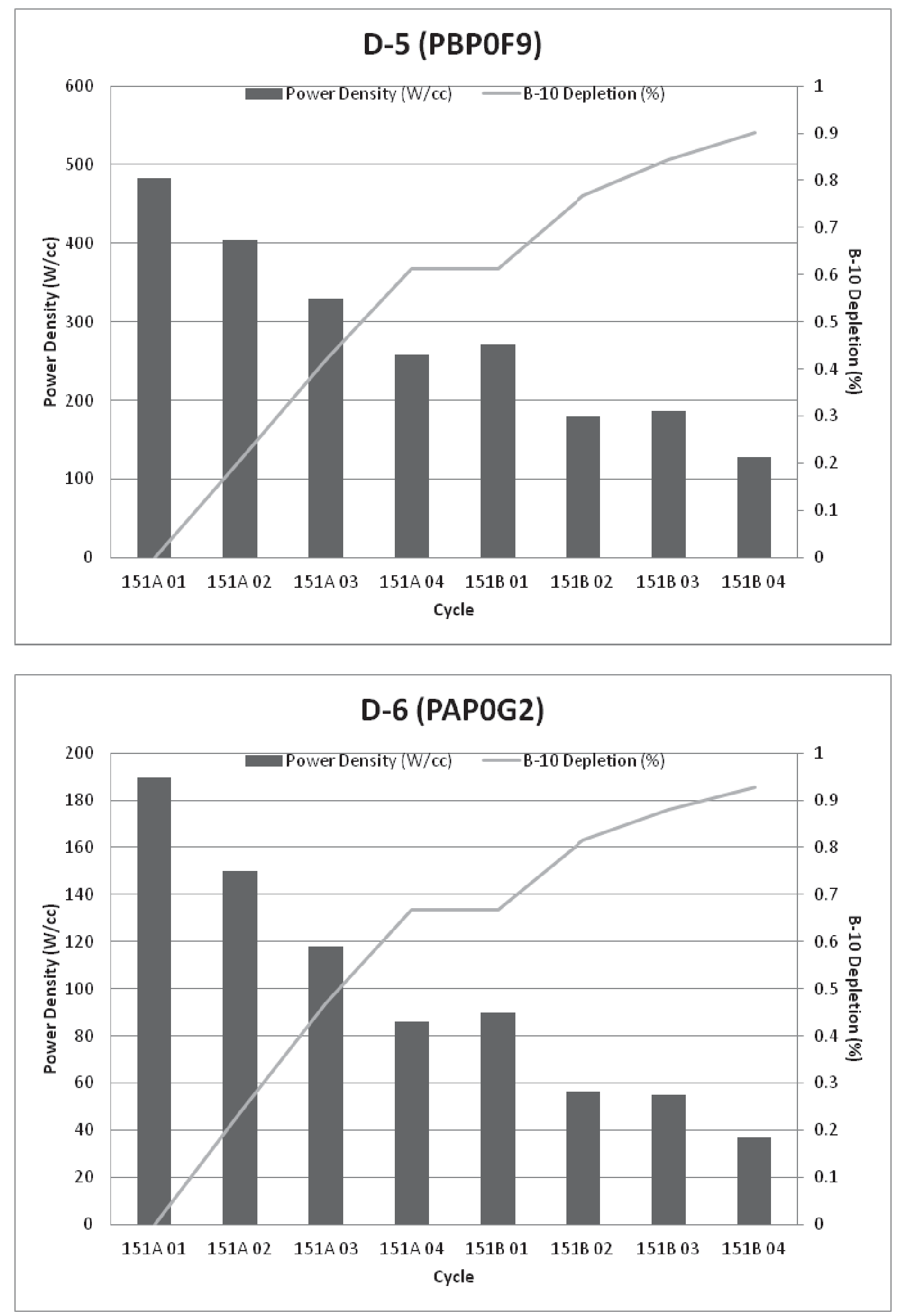

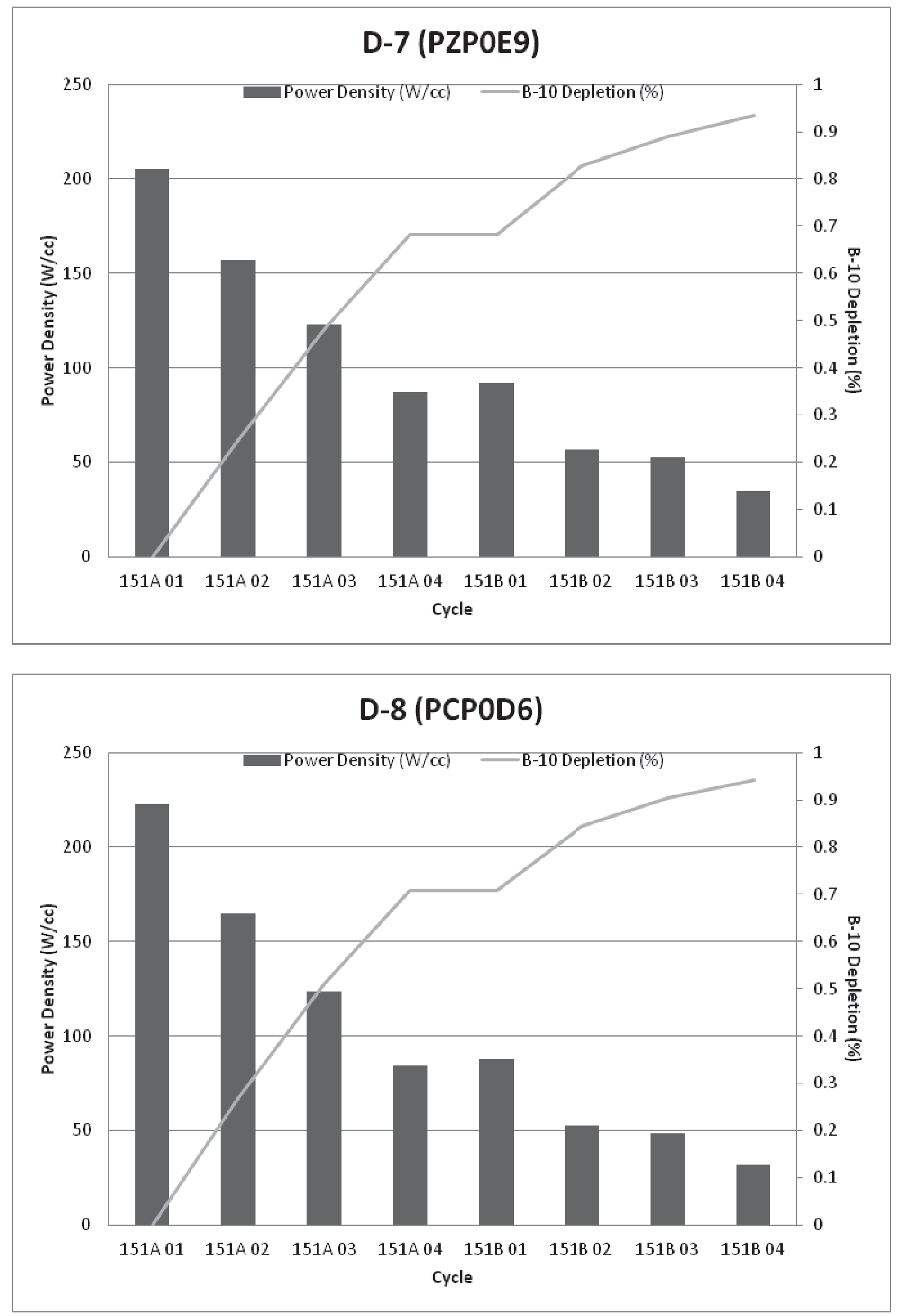